TRANSACTIONS OF THE

AMERICAN MATHEMATICAL SOCIETY

Volume 365, Number 2, February 2013, Pages 911-938

S 0002-9947(2012)05646-7

Article electronically published on August 9, 2012

\title{
HYPERBOLIC COMPOSITION OPERATORS ON THE BALL
}

\author{
F. BAYART AND S. CHARPENTIER
}

\begin{abstract}
We give a classification, up to automorphisms, of hyperbolic linear fractional maps of the ball. We then show that this classification is very convenient to study the geometric properties of these maps, as well as the spectrum and the dynamics of the associated composition operators. We conclude by showing how these properties can be transfered to composition operators associated to hyperbolic self-maps of the ball which are not linear-fractional maps.
\end{abstract}

\section{INTRODUCTION}

If $X$ is a Banach space of holomorphic functions on a domain $\mathcal{U}$ and if $\phi$ is a (holomorphic) self-map of $\mathcal{U}$, the composition operator of symbol $\phi$ is defined by $C_{\phi}(f)=f \circ \phi$ for any $f \in X$. The study of composition operators consists in the comparison of the properties of the operator $C_{\phi}$ with that of the function $\phi$ itself, which is called the symbol of $C_{\phi}$. For instance, if $X$ is the Hardy space $H^{2}(\mathbb{D})$ on the unit disk $\mathbb{D}$, it is well known that every self-map $\phi$ induces a bounded composition operator on $H^{2}(\mathbb{D})$; one can characterize compactness of $C_{\phi}$ and many other properties. We refer to [11] and to [21] to learn much more on this subject.

Even on the disk, some properties are not easily managed. A strategy to study composition operators is to use the so-called linear fractional model. Recall that a linear fractional map of the Riemann sphere $\hat{\mathbb{C}}$ is a map $u: \hat{\mathbb{C}} \rightarrow \hat{\mathbb{C}}$ which can be written as $u(z)=\frac{a z+b}{c z+d}$. The linear fractional model theorem asserts that every univalent self-map of the disk is conjugate to a linear fractional self-map of some, usually more complicated, plane domain. It is particularly useful for composition operators because, if $\phi: \mathbb{D} \rightarrow \mathbb{D}$ is holomorphic and $u$ is its model, one can often deduce properties of $C_{\phi}$ from properties of $C_{u}$. Of course, it is easier to study $C_{u}$ directly.

On the other hand, the theory of composition operators on the (Euclidean) ball $\mathbb{B}_{d}:=\left\{Z=(z, w) \in \mathbb{C} \times \mathbb{C}^{d-1}:\|Z\|_{2}^{2}<1\right\}$ of $\mathbb{C}^{d}, d \geq 2$ is much more involved. There are simple examples of self-maps of $\mathbb{B}_{d}$ which do not induce a bounded composition operator on $H^{2}\left(\mathbb{B}_{d}\right)$. In this context, the introduction of the class of linear fractional maps of the ball seems to be crucial, at least for two reasons: first, to give a source of examples that are easily managed, yet still rich enough to exhibit surprisingly diverse behaviors; second, to hope to produce a tool such as the linear fractional model. This motivated Cowen and MacCluer to introduce in [12] the following definition.

Received by the editors June 15, 2010 and, in revised form, June 10, 2011.

2010 Mathematics Subject Classification. Primary 47B33; Secondary 32A35.

Key words and phrases. Composition operators, hypercyclicity, linear fractional maps, supercyclicity. 
Definition 1.1. An application $\phi: \mathbb{C}^{d} \rightarrow \mathbb{C}^{d}$ is said to be a linear fractional map if it can be written as

$$
\phi(Z)=\frac{A Z+B}{\langle Z, C\rangle+D},
$$

where $A \in \mathcal{M}_{d}(\mathbb{C})$ is a matrix, $B$ and $C$ are two vectors in $\mathbb{C}^{d}$, and $D \in \mathbb{C}$.

If $\mathcal{U}$ is a domain of $\mathbb{C}^{d}$, we say that $\phi$ is a linear fractional map of $\mathcal{U}$ if it is defined in a neighbourhood of $\mathcal{U}$ and if $\phi(\mathcal{U}) \subset \mathcal{U}$. We denote the set of such maps by $\operatorname{LFM}(\mathcal{U})$.

In order to classify these linear fractional maps according to their fixed points (which play an essential role in the study of the associated composition operators), we recall the Denjoy-Wolff theorem in the ball $\mathbb{B}_{d}$ :

Theorem 1.2. Let $\phi \in L F M\left(\mathbb{B}_{d}\right)$ without fixed points in $\mathbb{B}_{d}$. Then there exists a unique fixed point $\tau \in \partial \mathbb{B}_{d}$ such that $\phi(\tau)=\tau$ and $\alpha(\phi)=\left\langle d \phi_{\tau}(\tau), \tau\right\rangle \in(0,1]$.

$\tau$ is called the Denjoy-Wolff point of $\phi$ and $\alpha(\phi)$ is called the boundary dilation coefficient of $\phi$.

Looking at this theorem, we may distinguish three types of linear fractional map $\phi$ of $\mathbb{B}_{d}$ :

- $\phi$ is said to be elliptic if it has a fixed point in $\mathbb{B}_{d}$;

- $\phi$ is said to be parabolic if it has no fixed point in $\mathbb{B}_{d}$ and if $\alpha(\phi)=1$;

- $\phi$ is said to be hyperbolic if it has no fixed point in $\mathbb{B}_{d}$ and if $\alpha(\phi) \in(0,1)$.

From now on, if $\phi \in L F M\left(\mathbb{B}_{d}\right)$ has no fixed points in $\mathbb{B}_{d}$, then, up to conjugation by an automorphism of the ball, we may assume that the Denjoy-Wolff point of $\phi$ is $e_{1}=(1,0, \ldots, 0)$.

In this paper, we intend to begin a systematic study of hyperbolic linear fractional maps and of their associated composition operators. Our first task is to classify the hyperbolic linear fractional maps. We move to the Siegel upper halfspace

$$
\mathbb{H}_{d}=\left\{Z=(z, w) \in \mathbb{C} \times \mathbb{C}^{d-1}, \Im m(z)>\left|w_{1}\right|^{2}+\cdots+\left|w_{d-1}\right|^{2}=\|w\|^{2}\right\}
$$

and we show that any hyperbolic linear fractional map of $\mathbb{B}_{d}$ is conjugated, via the Cayley transform and automorphisms of $\mathbb{B}_{d}$, to a self-map of $\mathbb{H}_{d}$ which may be written as

$$
\psi(z, u, v)=(\lambda z+b, D u, A v+c)
$$

where $z \in \mathbb{C},(u, v) \in \mathbb{C}^{d-1}, \Im m(b) \geq 0, D$ is a diagonal matrix with diagonal coefficients having modulus $\sqrt{\lambda}$ and $A$ and $c$ satisfy several technical conditions. We call this map $\psi$ a normal form of $\phi$, and this rewriting of $\phi$ will allow us to simplify the computations in the next parts of the paper.

Although the normal form is not unique up to automorphisms, we shall identify three properties of $\phi$ which may be easily read on any of its normal forms and which are invariant by automorphisms. We will call these properties the signature of $\phi$, and we shall see that the properties of both $\phi$ and $C_{\phi}$ do really depend on the signature. This will be for instance the case for the geometric properties of $\phi$, such as the shape of its characteristic domain, which is entirely determined by the signature of $\phi$.

Next, we will focus on the composition operators associated to hyperbolic linear fractional maps, and first of all, on their spectra. The study of the spectrum of 
composition operators has drawn the attention of many mathematicians; see for instance [11, Chapter 7]. In our context of linear fractional maps, the spectrum of $C_{\phi}$ has been computed when $\phi$ is parabolic in [2] and when $\phi$ is an automorphism (see [18]). The spectral radius is also known (see [17]).

We achieve the computation for hyperbolic linear fractional maps.

Theorem 1.3. Let $\phi \in \operatorname{LFM}\left(\mathbb{B}_{d}\right)$ be hyperbolic and not an automorphism. Then the spectrum of $C_{\phi}$ is

- $\bigcup_{n} C_{n} \cup\{0\}$, where $\left(C_{n}\right)$ is a sequence of coronae centered at 0 whose radii go to zero, if $\phi$ acts as an automorphism on a slice of $\mathbb{B}_{d}$;

- a disk centered at 0 otherwise.

In Section 4, we do a complete study of the dynamics of the composition operator associated to a hyperbolic linear fractional map. Recall that an operator $T$ acting on a Banach space $X$ is called hypercyclic if there exists a vector $x \in X$ such that its orbit $\left\{T^{n} x: n \geq 0\right\}$ is dense in $X$. It is called supercyclic if there exists a vector $x \in X$ such that its projective orbit $\mathbb{C} \cdot\left\{T^{n} x: n \geq 0\right\}$ is dense in $X$. It is called cyclic if there exists $x \in X$ such that $\{P(T) x: P$ polynomial $\}$ is dense in $X$. For a complete and up-to-date account on linear dynamics, we refer to [3].

The dynamics of composition operators is an important feature of both linear dynamics and the theory of composition operators (see for instance [2, 6], 9], 15], [16], 21]). In particular, the dynamics of a composition operator associated to a hyperbolic linear fractional map of the disk is already well known (see [6]):

Theorem 1.4 (Disk theorem). Let $\phi \in L F M(\mathbb{D})$ be hyperbolic. Then $C_{\phi}$ is hypercyclic.

The corresponding theorem for $\mathbb{B}_{d}, d \geq 2$, is much more complicated.

Theorem 1.5. Let $\phi \in \operatorname{LFM}\left(\mathbb{B}_{d}\right)$ be hyperbolic and univalent and let $\psi(z, w)=$ $(\lambda z+b, D u, A v+c)$ be a normal form of $\phi$.

(1) If $\operatorname{dim}(v)=0$, then $C_{\phi}$ is hypercyclic.

(2) If $\operatorname{dim}(v)>0$, then

(a) $C_{\phi}$ is never hypercyclic;

(b) $C_{\phi}$ is supercyclic iff $\Im m(b)>0$;

(c) $C_{\phi}$ is always cyclic.

This result is, for at least two reasons, rather surprising. First of all, we get examples of supercyclic composition operators which are not hypercyclic. This seems to be the first such examples and, indeed, it has been proved in 4 that in some cases (of course, not in this one!), compositional supercyclicity implies compositional hypercyclicity. Second, that $C_{\phi}$ has better dynamical properties when $\Im m(b)>0$ is not intuitive. The condition $\Im m(b)=0$ means that $z \mapsto$ $\lambda z+b$ is an automorphism of the upper half-plane (that the restriction of $\phi$ to some slice of $\mathbb{B}_{d}$ is an automorphism). Generally, the dynamical properties of a composition operator are better when $\phi$ is an automorphism in contradiction which what happens here. We shall prove Theorem 1.5 in Section 4 . This will depend heavily on the work done before.

Finally, we end the paper by applying the results on hyperbolic linear fractional composition operators to get results on general hyperbolic composition operators via the linear fractional model. As mentioned before, this technique was very 
efficient in the disk setting, especially for the dynamics of composition operators; see [6]. A linear fractional model for hyperbolic maps of the ball has been developed in [1] and in 8]. Due to new difficulties which arise in the several-variables setting, we will not obtain a result as general as that of [6]. However, the theorem that we will get is hopeful because it shows that the study of composition operators associated to linear fractional maps does have applications to general composition operators, even on $\mathbb{B}_{d}$ with $d \geq 2$. Thus the idea of Cowen and MacCluer was justified.

Notation. In what follows, the notation $f(x) \lesssim g(x)$ means that there exists some $C>0$ such that, for any $x, f(x) \leq C g(x)$. $\mathbb{P}_{+}$denotes the upper half-plane, $\mathbb{P}_{+}=\{z \in \mathbb{C}: \Im m(z)>0\}$.

\section{Geometric study}

2.1. Moving to the Siegel half-space. Let $\phi \in L F M\left(\mathbb{B}_{d}\right)$ without fixed points in $\mathbb{B}_{d}$ and whose Denjoy-Wolff point is $e_{1}$. The geometric properties of $C_{\phi}$ will be easier to understand if we move to the Siegel upper half-space, defined as follows:

Definition 2.1. The Siegel upper half-space $\mathbb{H}_{d}$ of $\mathbb{C}^{d}$ is defined by

$$
\mathbb{H}_{d}=\left\{(z, w) \in \mathbb{C} \times \mathbb{C}^{d-1}, \Im m(z)>\left|w_{1}\right|^{2}+\ldots+\left|w_{d-1}\right|^{2}=\|w\|^{2}\right\} .
$$

As in the complex plane, there exists a biholomorphism $\sigma_{c}$ from $\mathbb{B}_{d}$ onto $\mathbb{H}_{d}$ called the Cayley transform and given by the following formula:

$$
\sigma_{c}(z, w)=\left(i \frac{1+z}{1-z}, \frac{i w}{1-z}\right),(z, w) \in \mathbb{C} \times \mathbb{C}^{d-1} .
$$

Its reciprocal satisfies

$$
\sigma_{c}^{-1}(z, w)=\left(\frac{z-i}{z+i}, \frac{2 w}{z+i}\right) .
$$

This map may be extended to $\overline{\mathbb{B}_{d}}$ and it is then onto $\mathbb{H}_{d} \cup \partial \mathbb{H}_{d} \cup\{\infty\}$, where $\sigma_{c}(1)=\infty$.

Since the Cayley map is itself a linear fractional map, it is clear that $\operatorname{LFM}\left(\mathbb{H}_{d}\right)=$ $\sigma_{c}^{-1} \circ L F M\left(\mathbb{B}_{d}\right) \circ \sigma_{c}$. Moreover, if $\phi \in L F M\left(\mathbb{B}_{d}\right)$ admits $e_{1}$ as a Denjoy-Wolff point, the Denjoy-Wolff point of $\psi=\sigma_{c}^{-1} \circ \phi \circ \sigma_{c}$ is $\infty$.

It will be technically easier to deal with linear fractional maps of $\mathbb{H}_{d}$ fixing $\infty$ because the fractional part disappears for these maps. Precisely, F. Bracci, M. D. Contreras, S. Diaz-Madrigal in [7] have computed which maps of $\operatorname{LFM}\left(\mathbb{H}_{d}\right)$ fix $\infty$.

Theorem 2.2. Let $\psi=\sigma_{c}^{-1} \circ \phi \circ \sigma_{c} \in L F M\left(\mathbb{H}_{d}\right)$ be non-elliptic with DenjoyWolff point $\infty$ and let $\lambda=1 / \alpha(\phi)$. Then there exist $a, c \in \mathbb{C}^{d-1}, b \in \mathbb{C}$ and $M=M_{\phi} \in \mathcal{M}_{d-1}(\mathbb{C})$, such that

$$
\psi(z, w)=(\lambda z+2 i\langle w, a\rangle+b, M w+c) .
$$

Moreover, an application $\psi$ satisfying (2) maps $\mathbb{H}_{d}$ into itself if and only if

(a) $Q=\lambda I-M M^{*}$ is a Hermitian positive semi-definite matrix;

(b) $\Im m(b)-\|c\|^{2} \geq\left\langle Q^{+}\left(M^{*} c-a\right), M^{*} c-a\right\rangle$, where $Q^{+}$is the pseudo-inverse of $Q$

(c) $M^{*} c-a$ belongs to the space spanned by the columns of $Q$. 
In the present paper, we intend to study more specifically the hyperbolic linear fractional maps. We begin by simplifying, up to conjugation by automorphisms, formula (2).

2.2. Normal form of a hyperbolic linear fractional map. Let $\phi \in L F M\left(\mathbb{B}_{d}\right)$ be of hyperbolic type (with Denjoy-Wolff point at $e_{1}$ ) and let $\psi=\sigma_{c}^{-1} \circ \phi \circ \sigma_{c} \in$ $\operatorname{LFM}\left(\mathbb{H}_{d}\right)$ be the conjugated map in the Siegel half-space. Let us recall that $\lambda=1 / \alpha(\phi)>1$. Property (a) of Theorem 2.2 entails that $\|M\| \leq \sqrt{\lambda}$, so that the spectrum of $M$ is contained in the closed disk of radius $\sqrt{\lambda}$. Moreover, the same property also yields that the characteristic subspaces of $M$ associated to the eigenvalues of modulus $\sqrt{\lambda}$ coincide with the corresponding eigenvectorspaces, and that their orthogonal subspace is invariant by $M$. As a consequence, conjugating $M$ by a unitary map $U$, which means that we conjugate $\psi$ by the automorphism of $\mathbb{H}_{d}(z, w) \mapsto(z, U w)$, we may assume that the matrix $M$ which appears in (2) may be written as

$$
M=\left(\begin{array}{cc}
D & 0 \\
0 & A
\end{array}\right)
$$

where $D$ is a diagonal matrix whose diagonal coefficients all have modulus equal to $\sqrt{\lambda}$, and where the matrix $A$ is such that $\lambda I-A A^{*}$ is a Hermitian positive definite matrix.

Continuing in this way, we now conjugate $\psi$ by an automorphic Heisenberg translation $\tau$ of the Siegel half-space,

$$
\tau(z, w)=(z+2 i\langle w, \gamma\rangle+\beta, w+\gamma)
$$

with $\Im m(\beta)=\|\gamma\|^{2}$. We have (see [2])

$$
\tau^{-1}(z, w)=\left(z-2 i\langle w, \gamma\rangle-\beta+i\|\gamma\|^{2}, w-\gamma\right) .
$$

After a small computation, one obtains

$\tau^{-1} \circ \psi \circ \tau(z, w)=\left(\lambda z+2 i\left\langle w,\left(\bar{\lambda}-M^{*}\right) \gamma+a\right\rangle+b+2 i\langle M \gamma, \gamma\rangle+\lambda \beta, M(w+\gamma)+c\right)$.

Since $\left\|M^{*}\right\| \leq \sqrt{\lambda}$ and $\lambda>1, \bar{\lambda}-M^{*}$ is invertible and we choose $\gamma$ such that $\left(\lambda-M^{*}\right)(\gamma)=-a$. Next, adjusting the value of $\Re e(\beta)$, which is independent of $\|\gamma\|^{2}$, we may be sure that $b+2 i\langle M \gamma, \gamma\rangle+\lambda \beta$ is pure imaginary.

Therefore, up to conjugation by automorphisms, we get the following reduced formula for $\psi \in L F M\left(\mathbb{H}_{d}\right)$ hyperbolic:

$$
\psi(z, w)=\left(\lambda z+b, D u+c_{1}, A v+c_{2}\right),
$$

where $b$ is pure imaginary, and $w=(u, v)$ and $c=\left(c_{1}, c_{2}\right)$ are respectively the decompositions of $w$ and of $c$ along the orthogonal subspaces of $\mathbb{C}^{d-1}$ involved in the decomposition of $M$ in (3). Besides, as $\psi$ verifies condition (드, $M^{*} c$ belongs to the space spanned by the columns of $Q=\lambda I-M^{*} M=\left(\begin{array}{cc}0 & 0 \\ 0 & \lambda I-A^{*} A\end{array}\right)$, so that we must have $c_{1}=0$ because $D^{*}$ is one-to-one. Moreover, if $\Im m(b)=0$, then $c_{2}$ is also equal to 0 by (b).

We now conjugate $\psi$ by a non-isotropic dilation $h_{\mu}$,

$$
h_{\mu}(z, w)=(\mu z, \sqrt{\mu} w), \mu>0 .
$$

We get

$$
h_{\mu} \circ \psi \circ h_{1 / \mu}(z, w)=(\lambda z+\mu b, D u, A v+\sqrt{\mu} c) .
$$


In particular, when $\Im m(b)>0$, we may choose $\mu$ such that $\Im m(\mu b)$ is very close to 0 . For our applications, we will need $\Im m(\mu b) \in(0, \lambda-1)$.

Finally, any matrix is always unitarily conjugated to an upper-triangular matrix. This means that, up to automorphisms, we may assume that the matrix $A$ is uppertriangular. We summarize the work done until now in the following proposition:

Proposition 2.3. Let $\phi \in \operatorname{LFM}\left(\mathbb{B}_{d}\right)$ be hyperbolic and let $\psi=\sigma_{c}^{-1} \circ \phi \circ \sigma_{c}$. Then $\psi$ is conjugated to a map

$$
(z, w) \mapsto(\lambda z+b, D u, A v+c),
$$

where

(a) $D$ is diagonal with diagonal coefficients of modulus $\sqrt{\lambda}$;

(b) $Q=\lambda I-A^{*} A$ is Hermitian positive definite and $A$ is upper-triangular;

(c) $b$ is pure imaginary and $\|c\|^{2}+\left\langle Q^{-1} A^{*} c, A^{*} c\right\rangle \leq \Im m(b)<\lambda-1$. In particular, if $b$ is equal to zero, then $c$ is equal to zero.

The map appearing in (4) will be called a normal form of $\phi$.

A linear fractional map of $\mathbb{B}_{d}$ does not admit a unique normal form. However, several parameters of it are invariant by conjugation by automorphisms fixing $\infty$ :

- $\lambda_{\phi}:=\lambda=1 / \alpha(\phi)$, which is the inverse of the boundary dilation coefficient of $\phi$;

- the fact that $\Im m(b)=0$ or not. This determines whether $\psi_{1}$ is, or is not, an automorphism of $\{\Im m(z)>0\}$. Coming back to $\phi$, $\Im m(b)=0$ if and only if $\phi_{\mid D}$ acts as an automorphism on some slice $D$ of $\mathbb{B}_{d}$. We set $\varepsilon_{\phi}=1$ if $\Im m(b)=0, \varepsilon_{\phi}=0$ otherwise;

- the number of eigenvalues of $M$, counted with their multiplicity, which have modulus $\sqrt{\lambda}$. We shall denote by $p_{\phi}$ this number of eigenvalues, namely the dimension of $u$.

Definition 2.4. The triple $\left(\lambda_{\phi}, \varepsilon_{\phi}, p_{\phi}\right)$ is called the signature of $\phi$.

The signature classifies, up to conjugation by automorphisms, the linear fractional maps of $\mathbb{B}_{d}$. Our intention is to convince the reader that, as soon as we know the signature of $\phi$, we know many things on the behavior of $\phi$ and on its associated composition operator. We begin by some geometrical considerations.

2.3. Characteristic domain of a hyperbolic type linear fractional map. We recall that the characteristic domain of $\phi \in L F M\left(\mathbb{B}_{d}\right)$ is the smallest domain containing the ball and invariant by $\phi$. When $\phi$ is univalent (when $M_{\phi}$ is one-toone), this is the smallest domain containing $\mathbb{B}_{d}$ on which $\phi$ acts as an automorphism.

Theorem 2.5. Let $\phi \in L F M\left(\mathbb{B}_{d}\right)$ be hyperbolic and let $\psi(z, w)=(\lambda z+b, D u, A v+$ c) be a normal form of $\phi$. Then the characteristic domain of $\phi$ is, up to conjugation by the Cayley transform and by automorphisms of $\mathbb{H}_{d}$, equal to

$$
\Omega_{0}:=\left\{(z, u, v) \in \mathbb{C}^{d}, \Im m(z)>\|u\|^{2}-\frac{\Im m(b)}{\lambda-1}\right\} .
$$

Proof. The characteristic domain of $\psi$ is $\Omega=\bigcup_{n \geq 0} \psi^{-n}\left(\mathbb{H}_{d}\right)$. It suffices to prove that $\Omega$ is equal to $\Omega_{0}$. Let $\psi_{n}$ denotes the $n^{\text {th }}$ iteration of $\psi$. A straightforward computation gives

$$
\psi_{n}(z, w)=\left(\lambda^{n} z+\frac{\lambda^{n}-1}{\lambda-1} b, D^{n} u, A^{n} v+A^{n-1} c+\cdots+c\right)
$$


so that $(z, w) \in \bigcup_{n \geq 0} \psi^{-n}\left(\mathbb{H}_{d}\right)$ iff there exists an integer $n$ such that

$$
\begin{aligned}
& \Im m(z)+\frac{1}{\lambda^{n}}\left(\frac{\lambda^{n}-1}{\lambda-1}\right) \Im m(b)>\|u\|^{2}+\frac{1}{\lambda^{n}}\left\|A^{n} v+A^{n-1} c+\cdots+c\right\|^{2} \\
& \Longleftrightarrow \Im m(z)>\|u\|^{2}-\frac{\Im m(b)}{\lambda-1}+\frac{\Im m(b)}{\lambda^{n}(\lambda-1)}+\frac{1}{\lambda^{n}}\left\|A^{n} v+A^{n-1} c+\cdots+c\right\|^{2} .
\end{aligned}
$$

This shows immediately that $\Omega \subset \Omega_{0}$. The converse inclusion is also satisfied since

$$
\left\{\begin{array}{l}
\frac{\Im m(b)}{\lambda^{n}(\lambda-1)} \rightarrow 0 \\
\frac{1}{\lambda^{n}}\left\|A^{n} v+A^{n-1} c+\cdots+c\right\|^{2} \rightarrow 0
\end{array}\right.
$$

(recall that $\|A\| \leq \sqrt{\lambda}$ ).

We notice that, if we want to know the shape of the characteristic domain, the important part of the signature of $\phi$ is the value of $p_{\phi}$.

2.4. Convergence to infinity. Let $\psi$ be a self-map of $\mathbb{H}_{d}$ with Denjoy-Wolff point at $\infty$. For each $(z, w) \in \mathbb{H}_{d}, \psi_{n}(z, w)$ goes to $\infty$. Two types of convergence (which are invariant by conjugation by an automorphism) are of particular interest:

Definition 2.6. Given $\left(z_{n}, w_{n}\right)$ a sequence of points in $\mathbb{H}_{d}$ convergent to infinity. We say that this convergence is

(a) special if $\frac{\left\|w_{n}\right\|^{2}}{\Im m\left(z_{n}\right)}$ tends to 0 ;

(b) restrictive if $\left(z_{n}\right)_{n}$ converges non-tangentially to infinity, that is to say, there exists $C>0$ such that $\left|\Re e\left(z_{n}\right)\right| \leq C \Im m\left(z_{n}\right)$ for any $n \geq 0$.

The classification of hyperbolic linear fractional maps gives the following result:

Theorem 2.7. Let $\phi \in L F M\left(\mathbb{B}_{d}\right)$ be hyperbolic and let $\psi=\sigma_{c}^{-1} \circ \phi \circ \sigma_{c}$ be its conjugated map in $\mathbb{H}_{d}$. For any $(z, u, v) \in \mathbb{H}_{d}, \psi_{n}(z, u, v)$ converges to its DenjoyWolff point $\infty$ in a restrictive way. This convergence is special if and only if $u=0$. In particular, every orbit is special if and only if $p_{\phi}=0$.

Proof. Since special and restrictive convergence are invariant by conjugation by an automorphism, we may assume that $\psi$ is a normal form of $\phi$. Put $\left(z_{n}, w_{n}\right):=$ $\psi_{n}(z, w) \in \mathbb{H}_{d}$. Since the Denjoy-Wolff point of $\psi$ is $\infty,\left(z_{n}\right)$ must tend to infinity. Moreover

$$
\frac{\left|\Re e\left(z_{n}\right)\right|}{\Im m\left(z_{n}\right)}=\frac{\lambda^{n} \Re e(z)}{\lambda^{n} \Im m(z)+\frac{\lambda^{n}-1}{\lambda-1} \Im m(b)} \underset{n \rightarrow \infty}{\longrightarrow} \frac{\Re e(z)}{\Im m(z)+\frac{\Im m(b)}{\lambda-1}},
$$

proving the restrictive convergence.

Besides,

$$
\frac{\left\|w_{n}\right\|^{2}}{\Im m\left(z_{n}\right)}=\frac{\left\|D^{n} u\right\|^{2}+\left\|A^{n} v+A^{n-1} c+\cdots+c\right\|^{2}}{\lambda^{n} \Im m(z)+\frac{\lambda^{n}-1}{\lambda-1} \Im m(b)} \underset{n \rightarrow \infty}{\longrightarrow} \frac{\|u\|^{2}}{\Im m(z)+\frac{\Im m(b)}{\lambda-1}},
$$

so that the convergence to infinity is special if and only if the subspace associated to the eigenvalues of modulus $\sqrt{\lambda}$ in the normal form of $\psi$ is reduced to 0 . 


\section{Spectrum of hyperbolic COMposition Operators}

Let $\phi \in \operatorname{LFM}\left(\mathbb{B}_{d}\right)$ be hyperbolic. We suppose that $\phi$ is not an automorphism of $\mathbb{B}_{d}$ and we intend to compute the spectrum of $C_{\phi}$. We first recall the following result of M. Jury, which gives the spectral radius:

Theorem 3.1 ([17, Corollary 13]). If $\phi$ is a hyperbolic linear fractional map of the ball $\mathbb{B}_{d}$ with dilation coefficient $\alpha$, then the spectral radius of $C_{\phi}$ acting on $H^{2}\left(\mathbb{B}_{d}\right)$ is $\alpha^{-d / 2}$.

As one can guess, our main tool to prove Theorem 1.3 will be the normal form of $\phi$. Precisely, if $\psi$ denotes a normal form of $\phi$, we will compute the spectrum of $C_{\psi}$. To do that, we have to define the space where $C_{\psi}$ acts. The image of $H^{2}\left(\mathbb{B}_{d}\right)$ by the Cayley transform is denoted by $\mathcal{H}^{2}\left(\mathbb{H}_{d}\right)$ :

$$
\mathcal{H}^{2}\left(\mathbb{H}_{d}\right)=\left\{F: \mathbb{H}_{d} \rightarrow \mathbb{C} \text { holomorphic; } F \circ \sigma_{c} \in H^{2}\left(\mathbb{B}_{d}\right)\right\} \text {. }
$$

$\mathcal{H}^{2}\left(\mathbb{H}_{d}\right)$ is endowed with the norm inherited from $H^{2}\left(\mathbb{B}_{d}\right) ;\|F\|_{\mathcal{H}^{2}}=\left\|F \circ \sigma_{c}\right\|_{H^{2}}$ so that $C_{\sigma_{c}}$ is a unitary map from $\mathcal{H}^{2}\left(\mathbb{H}_{d}\right)$ onto $H^{2}\left(\mathbb{B}_{d}\right)$. As one easily sees by computing the Jacobian of $\sigma_{c}$, the norm on $\mathcal{H}_{2}\left(\mathbb{H}_{d}\right)$ satisfies

$$
\|F\|_{\mathcal{H}^{2}}^{2}=\kappa^{2} \int_{\partial \mathbb{H}_{d}} \frac{|F(z, w)|^{2}}{|z+i|^{2 d}} d \sigma_{\partial \mathbb{H}_{d}}
$$

where $\kappa$ is a constant that we will not try to compute and $d \sigma_{\partial \mathbb{H}_{d}}$ is the Lebesgue measure on $\partial \mathbb{H}_{d}$.

Of course, $C_{\phi}$ acting on $H^{2}\left(\mathbb{B}_{d}\right)$ and $C_{\psi}$ acting on $\mathcal{H}^{2}\left(\mathbb{H}_{d}\right)$ are unitarily similar, so that they share the same properties. We are now ready for the proof.

3.1. Spectrum when $\phi$ does not act as an automorphism on a slice. In this subsection, we compute the spectrum of $C_{\phi}$ when $\varepsilon_{\phi}=0$, namely when a normal form of $\phi$ is $\psi(z, w)=(\lambda z+b, D u, A v+c)$ with $\Im m(b)>0$.

Theorem 3.2. With the notation above, $\sigma\left(C_{\phi}\right)=\bar{D}\left(0, \lambda^{d / 2}\right)$.

Proof. We already know by Theorem 3.1 that $\sigma\left(C_{\phi}\right)$ is contained in the closed disk $\bar{D}\left(0, \lambda^{d / 2}\right)$. We need only to show that any element of $D\left(0, \lambda^{d / 2}\right) \backslash\{0\}$ is an eigenvalue of $C_{\phi}$. Now, given $s \in \mathbb{C}$, let us define

$$
F_{s}(z)=\left(z+\frac{b}{\lambda-1}\right)^{s}
$$

It is clear that $F_{s} \circ \psi=\lambda^{s} F_{s}$, and so we are interested in the values of $s$ such that the $F_{s}$ belong to $\mathcal{H}^{2}\left(\mathbb{H}_{d}\right)$. Now, setting $i t=b /(\lambda-1)$ with $t>0$, one gets

$$
\left\|F_{s}\right\|^{2}=\int_{\partial \mathbb{H}_{d}} \frac{\left|(z+i t)^{s}\right|^{2}}{|z+i|^{2 d}} d \sigma_{\partial \mathbb{H}_{d}} \lesssim \int_{\partial \mathbb{H}_{d}} \frac{1}{|z+i|^{2(d-\Re e(s))}} d \sigma_{\partial \mathbb{H}_{d}},
$$

and this last integral is finite iff $\Re e(s)<d / 2$. Hence, any $\lambda^{s}$ with $\Re e(s)<d / 2$ is an eigenvalue, achieving the proof of the theorem.

3.2. Spectrum when $\phi$ acts as an automorphism on a slice - Introduction. We are now in the situation where a normal form of $\phi$ is given by $\psi(z, w)=$ $(\lambda z, D u, A v)$. The previous proof does not work as well, because the functions 
$F_{s}(z)=z^{s}$ which satisfy $F_{s} \circ \psi=\lambda^{s} F_{s}$ belong to $\mathcal{H}^{2}\left(\mathbb{H}_{d}\right)$ if and only if $-d / 2<$ $\Re e(s)<d / 2$, showing only that the corona $\left\{\lambda^{-d / 2}<\Re e(s)<\lambda^{d / 2}\right\}$ is contained in $\sigma_{p}\left(C_{\phi}\right)$. This is sufficient to determine the spectrum of $C_{\phi}$ when $\phi$ is an automorphism of $\mathbb{B}_{d}$. Otherwise, this just gives the first corona which appears in Theorem 1.3. To get the remaining part of the spectrum, we have to introduce a nice decomposition of $\mathcal{H}^{2}\left(\mathbb{H}_{d}\right)$.

For $\alpha \in \mathbb{N}^{s}$ (here $s=d-1-p_{\phi}$ ), we denote by $\mathcal{H}_{\alpha}$ the set of all functions $F$ of $\mathcal{H}^{2}\left(\mathbb{H}_{d}\right)$ which can be written $F(z, u, v)=F_{\alpha}(z, u) v^{\alpha}$, where $v^{\alpha}$ stands for $\prod_{i=1}^{s} v_{i}^{\alpha_{i}}$. It is clear that $\mathcal{H}^{2}\left(\mathbb{H}_{d}\right)=\bigoplus_{\alpha \in \mathbb{N}^{s}}^{\perp} \mathcal{H}_{\alpha}$. Moreover, let us order $\mathbb{N}^{s}$ as follows:

$$
\alpha \prec \beta \text { iff }\left\{\begin{array}{l}
|\alpha|<|\beta| \\
\text { or } \\
|\alpha|=|\beta| \text { and there exists } j \text { such that } \alpha_{1}=\beta_{1}, \ldots, \alpha_{j-1}=\beta_{j-1} \text { and } \alpha_{j}<\beta_{j} .
\end{array}\right.
$$

If we define

$$
\mathcal{K}_{N}:=\bigoplus_{\substack{\alpha \in \mathbb{N}^{s} \\|\alpha| \geq N}}^{\perp} \mathcal{H}_{\alpha}
$$

then $C_{\phi}$ is upper triangular under the decomposition

$$
\mathcal{H}^{2}\left(\mathbb{H}_{d}\right)=\bigoplus_{\substack{\alpha \in \mathbb{N}^{s} \\|\alpha|<N}}^{\perp} \mathcal{H}_{\alpha} \oplus^{\perp} \mathcal{K}_{N}
$$

with respect to the order $\prec$ on $\mathbb{N}^{s}$ (recall that $A$ has been supposed to be upper triangular) and is diagonal if $A$ itself is diagonal. Finally let $T_{\alpha}$ be the diagonal block of $C_{\phi}$ corresponding to the subspace $\mathcal{H}_{\alpha} . T_{\alpha}$ is also the diagonal block corresponding to $\mathcal{H}_{\alpha}$ of the simpler composition operator associated to the application $\tilde{\psi}$ defined by

$$
\tilde{\psi}(z, u, v)=(\lambda z, D u, \tilde{A} v),
$$

where $\tilde{A}$ is the diagonal matrix composed of the eigenvalues of $A$. That $\tilde{\psi}$ is a self-map of $\mathbb{H}_{d}$ follows from the easily verified fact that $\|\tilde{A}\| \leq\|A\|$.

With this in mind, a general result concerning the spectrum of triangular or diagonal operators on a finite sum of subspaces of a given vector space (see for instance [2, Lemma 5.3]) directly yields the following lemma:

Lemma 3.3. With the previous notation, we have:

$$
\sigma\left(C_{\psi}\right) \subseteq \bigcup_{\substack{\alpha \in \mathbb{N}^{s} \\|\alpha|<N}} \sigma\left(T_{\alpha}\right) \cup \overline{D\left(0,\left\|C_{\psi \mid \mathcal{K}_{N}}\right\|\right)} .
$$

In view of Lemma 3.3, our strategy is now rather clear:

Step 1: Show that $\left\|C_{\psi \mid \mathcal{K}_{N}}\right\|$ goes to zero as $N$ goes to $\infty$. By the above lemma, this will yield

$$
\sigma\left(C_{\psi}\right) \subset \bigcup_{\alpha \in \mathbb{N}^{s}} \sigma\left(T_{\alpha}\right) \cup\{0\} .
$$


Step 2: Using the operator $C_{\tilde{\psi}}$, which is diagonal with diagonal blocks $T_{\alpha}$, we will prove that

$$
\sigma\left(T_{\alpha}\right) \subset\left\{z \in \mathbb{C}, \lambda^{-d / 2} \prod_{i=1}^{s}\left(\frac{\left|\mu_{i}\right|}{\sqrt{\lambda}}\right)^{\alpha_{i}} \leq|z| \leq \lambda^{d / 2} \prod_{i=1}^{s}\left(\frac{\left|\mu_{i}\right|}{\sqrt{\lambda}}\right)^{\alpha_{i}}\right\}
$$

where $\mu_{1}, \ldots, \mu_{s}$ are the eigenvalues of $A$, written with multiplicity.

Step 3: For each $\alpha \in \mathbb{N}^{s}$ and each $z \in \mathbb{C}$ belonging to

$$
\left\{z \in \mathbb{C}, \lambda^{-d / 2} \prod_{i=1}^{s}\left(\frac{\left|\mu_{i}\right|}{\sqrt{\lambda}}\right)^{\alpha_{i}}<|z|<\lambda^{d / 2} \prod_{i=1}^{s}\left(\frac{\left|\mu_{i}\right|}{\sqrt{\lambda}}\right)^{\alpha_{i}}\right\}
$$

we shall exhibit an eigenvector of $C_{\psi}$ corresponding to the eigenvalue $z$.

Provided that we are able to do that, we obtain the following precise version of Theorem 1.3.

Theorem 3.4. Let $\phi \in L F M\left(\mathbb{B}_{d}\right)$ be such that $\varepsilon_{\phi}=1$ and let $\psi(z, w)=(\lambda z, D u, A v)$ be a normal form of $\phi$. Also let $\mu_{1}, \ldots, \mu_{s}$ be the eigenvalues of $A$. Then

$$
\sigma\left(C_{\phi}\right)=\bigcup_{\alpha \in \mathbb{N}^{s}}\left\{z \in \mathbb{C}, \lambda^{-d / 2} \prod_{i=1}^{s}\left(\frac{\left|\mu_{i}\right|}{\sqrt{\lambda}}\right)^{\alpha_{i}} \leq|z| \leq \lambda^{d / 2} \prod_{i=1}^{s}\left(\frac{\left|\mu_{i}\right|}{\sqrt{\lambda}}\right)^{\alpha_{i}}\right\} \cup\{0\}
$$

Let us proceed with the details of the proof.

3.3. Spectrum when $\phi$ acts as an automorphism on a slice - Step 1. The aim of this step is the following lemma.

Lemma 3.5. Under the above conditions and notation,

$$
\left\|C_{\psi \mid \mathcal{K}_{N}}\right\| \underset{N \rightarrow \infty}{\longrightarrow} 0
$$

Proof. Let $A=U \Sigma V$ be a singular value decomposition of $A ; U$ and $V$ are two unitary maps and $\Sigma$ is the diagonal matrix whose diagonal terms are the square roots of the eigenvalues of $A A^{*}$ :

$$
\Sigma=\left[\begin{array}{ccc}
\left|\mu_{1}\right| & \cdots & 0 \\
\vdots & \ddots & \vdots \\
0 & \cdots & \left|\mu_{s}\right|
\end{array}\right] \text { with in particular }\left|\mu_{i}\right| \in[0, \sqrt{\lambda}) .
$$

Then we factorize $C_{\psi}$ as $C_{\psi}=C_{\tau_{1}} \circ C_{\psi_{\Sigma}} \circ C_{\tau_{2}}=C_{\tau_{2} \circ \psi_{\Sigma} \circ \tau_{1}}$, where

$$
\left\{\begin{aligned}
\tau_{1}:(z, w) & \mapsto(z, u, V v) \\
\psi_{\Sigma}:(z, w) & \mapsto(\lambda z, D u, \Sigma v) \\
\tau_{2}:(z, w) & \mapsto(z, u, U v)
\end{aligned}\right.
$$

As $\tau_{1}$ and $\tau_{2}$ are automorphic self-maps of $\mathbb{H}_{d}$ which preserve both $\mathcal{K}_{N}$ and $\mathcal{K}_{N}^{\perp}$, $\left\|C_{\psi \mid \mathcal{K}_{N}}\right\| \underset{N \rightarrow \infty}{\longrightarrow} 0$ will stand as soon as

$$
\left\|C_{\psi_{\Sigma} \mid \mathcal{K}_{N}}\right\| \underset{N \rightarrow \infty}{\longrightarrow} 0
$$


We introduce $\psi_{0}=(\lambda z, D u, \sqrt{\lambda} v) \cdot \psi_{0}$ is an automorphism of $\mathbb{H}_{d}$; hence $C_{\psi_{0}}$ is invertible on $\mathcal{H}^{2}\left(\mathbb{H}_{d}\right)$. For $F \in \mathcal{H}_{\alpha}, F(z, u, v)=F_{\alpha}(z, u) v^{\alpha}$, we have both

$$
\begin{aligned}
& C_{\psi_{0}}(F)=F_{\alpha}(\lambda z, D u) \sqrt{\lambda}^{|\alpha|} v^{\alpha}, \\
& C_{\psi_{\Sigma}}(F)=F_{\alpha}(\lambda z, D u)\left(\prod_{i=1}^{s}\left|\mu_{i}\right|^{\alpha_{i}}\right) v^{\alpha} .
\end{aligned}
$$

This yields

$$
C_{\psi_{\Sigma} \mid \mathcal{K}_{N}}=\mathcal{A} \circ C_{\psi_{0}}
$$

where $\mathcal{A}$ is the diagonal operator on $K_{N}=\bigoplus_{|\alpha| \geq N}^{\perp} \mathcal{H}_{\alpha}$ whose diagonal block corresponding to $\mathcal{H}_{\alpha}$ is $\prod_{i=1}^{s}\left(\frac{\left|\mu_{i}\right|}{\sqrt{\lambda}}\right)^{\alpha_{i}} I$. Therefore

$$
\left\|C_{\psi_{\Sigma} \mid \mathcal{K}_{N}}\right\| \leq\|\mathcal{A}\| \cdot\left\|C_{\psi_{0}}\right\| \text {. }
$$

Moreover,

$$
\|\mathcal{A}\|=\max _{\substack{\alpha \in \mathbb{N}^{s} \\|\alpha| \geq N}} \prod_{i=1}^{s}\left(\frac{\left|\mu_{i}\right|}{\sqrt{\lambda}}\right)^{\alpha_{i}} \leq\left(\frac{\max _{1 \leq i \leq s}\left|\mu_{i}\right|}{\sqrt{\lambda}}\right)^{N} .
$$

Consequently,

$$
\left\|C_{\psi_{\Sigma} \mid \mathcal{K}_{N}}\right\| \leq\left\|C_{\psi_{0}}\right\|\left(\frac{\max _{1 \leq i \leq s}\left|\mu_{i}\right|}{\sqrt{\lambda}}\right)^{N} \underset{N \rightarrow \infty}{\longrightarrow} 0
$$

since $\left|\mu_{i}\right|<\sqrt{\lambda}$ for all $i$.

3.4. Spectrum when $\phi$ acts as an automorphism on a slice - Step 2. The next proposition provides an upper bound for the spectrum of $T_{\alpha}$ :

Proposition 3.6. With the above conditions and notation,

$$
\sigma\left(T_{\alpha}\right) \subset\left\{z \in \mathbb{C}, \lambda^{-d / 2} \prod_{i=1}^{s}\left(\frac{\left|\mu_{i}\right|}{\sqrt{\lambda}}\right)^{\alpha_{i}} \leq|z| \leq \lambda^{d / 2} \prod_{i=1}^{s}\left(\frac{\left|\mu_{i}\right|}{\sqrt{\lambda}}\right)^{\alpha_{i}}\right\} .
$$

Proof. Let $P_{\alpha}$ denote the orthogonal projection on $\mathcal{H}_{\alpha}$. As $T_{\alpha}=C_{\tilde{\psi}} \circ P_{\alpha}$, one has to prove that

$$
\sigma\left(C_{\tilde{\psi} \mid \mathcal{H}_{\alpha}}\right) \subset\left\{z \in \mathbb{C}, \lambda^{-d / 2} \prod_{i=1}^{s}\left(\frac{\left|\mu_{i}\right|}{\sqrt{\lambda}}\right)^{\alpha_{i}} \leq|z| \leq \lambda^{d / 2} \prod_{i=1}^{s}\left(\frac{\left|\mu_{i}\right|}{\sqrt{\lambda}}\right)^{\alpha_{i}}\right\},
$$

where we recall that $\tilde{\psi}(z, u, v)=(\lambda z, D u, \tilde{A} v)$ with

$$
\tilde{A}=\left[\begin{array}{ccc}
\mu_{1} & \cdots & 0 \\
\vdots & \ddots & \vdots \\
0 & \cdots & \mu_{s}
\end{array}\right]
$$

As before, we introduce the automorphism of $\mathcal{H}^{2}\left(\mathbb{H}_{d}\right)$ defined by $\psi_{0}=$ $(\lambda z, D u, \sqrt{\lambda} v)$. It is already well known (see [18]) that

$$
\sigma\left(C_{\psi_{0}}\right)=\left\{z \in \mathbb{C}, \lambda^{-d / 2} \leq|z| \leq \lambda^{d / 2}\right\} .
$$


Therefore, as $\mathcal{H}_{\alpha}$ and $\mathcal{H}_{\alpha}^{\perp}$ are stable under $C_{\psi_{0}}$,

$$
\sigma\left(C_{\psi_{0 \mid \mathcal{H}_{\alpha}}}\right) \subset \sigma\left(C_{\psi_{0}}\right)=\left\{z \in \mathbb{C}, \lambda^{-d / 2} \leq|z| \leq \lambda^{d / 2}\right\} .
$$

Moreover, for $F \in \mathcal{H}_{\alpha}, F(z, u, v)=F_{\alpha}(z, u) v^{\alpha}$, we have both

$$
\begin{aligned}
C_{\psi_{0}}(F) & =F_{\alpha}(\lambda z, D u) \sqrt{\lambda} v^{|\alpha|} v^{\alpha}, \\
C_{\tilde{\psi}}(F) & =F_{\alpha}(\lambda z, D u)\left(\prod_{i=1}^{s} \mu_{i}^{\alpha_{i}}\right) v^{\alpha},
\end{aligned}
$$

so that $C_{\tilde{\psi} \mid \mathcal{H}_{\alpha}}=\frac{\prod_{i=1}^{s} \mu_{i}^{\alpha_{i}}}{\sqrt{\lambda}^{|\alpha|}} C_{\psi_{0 \mid \mathcal{H}_{\alpha}}}$. This yields

$$
\begin{aligned}
\sigma\left(C_{\tilde{\psi}_{\mid \mathcal{H} \alpha}}\right) & =\frac{\prod_{i=1}^{s} \mu_{i}^{\alpha_{i}}}{\sqrt{\lambda}^{|\alpha|}} \sigma\left(C_{\psi_{0 \mid \mathcal{H}_{\alpha}}}\right) \\
& \subset\left\{z \in \mathbb{C}, \lambda^{-d / 2} \prod_{i=1}^{s}\left(\frac{\left|\mu_{i}\right|}{\sqrt{\lambda}}\right)^{\alpha_{i}} \leq|z| \leq \lambda^{d / 2} \prod_{i=1}^{s}\left(\frac{\left|\mu_{i}\right|}{\sqrt{\lambda}}\right)^{\alpha_{i}}\right\} .
\end{aligned}
$$

In fact, the inclusion appearing in the above proposition is an equality, as will be clear in the next subsection.

3.5. Spectrum when $\phi$ acts as an automorphism on a slice - Step 3. To end the proof of Theorem 3.4 , we just need the following proposition:

Proposition 3.7. With the above conditions and notation,

$\sigma_{p}\left(C_{\psi \mid \mathcal{H}_{\alpha}}\right) \supset\left\{z \in \mathbb{C}, \lambda^{-d / 2} \prod_{i=1}^{s}\left(\frac{\left|\mu_{i}\right|}{\sqrt{\lambda}}\right)^{\alpha_{i}}<|z|<\lambda^{d / 2} \prod_{i=1}^{s}\left(\frac{\left|\mu_{i}\right|}{\sqrt{\lambda}}\right)^{\alpha_{i}}\right\}$ for every $\alpha \in \mathbb{N}^{s}$.

In particular, we have

$$
\sigma_{p}\left(C_{\psi}\right) \supset \bigcup_{\alpha \in \mathbb{N}^{s}}\left\{z \in \mathbb{C}, \lambda^{-d / 2} \prod_{i=1}^{s}\left(\frac{\left|\mu_{i}\right|}{\sqrt{\lambda}}\right)^{\alpha_{i}}<|z|<\lambda^{d / 2} \prod_{i=1}^{s}\left(\frac{\left|\mu_{i}\right|}{\sqrt{\lambda}}\right)^{\alpha_{i}}\right\}
$$

Proof. For $t+i l \in \mathbb{C}$, let $F:(z, u, v) \longmapsto z^{t+i l} \tilde{v}(v)^{\alpha}$ with $\tilde{v}=(v(1), \ldots, v(s))$, where, for $1 \leq i \leq s, v(i) \in\left(\mathbb{C}^{s}\right)^{*}$ is the eigenvector of $A^{T}$ associated to the eigenvalue $\mu_{i}$ and $\tilde{v}^{\alpha}$ stands for $\tilde{v}^{\alpha}=\prod_{i=1}^{s} v(i)^{\alpha_{i}}$. We get

$$
C_{\psi}(F)=\lambda^{t+i l}\left(\prod_{i=1}^{s} \mu_{i}^{\alpha_{i}}\right) F
$$

Now, let us see for which values of $t+i l$ the function $F$ is in $\mathcal{H}^{2}\left(\mathbb{H}_{d}\right)$. Writing $v(i)(v)=a_{i, 1} v_{1}+\cdots+a_{i, s} v_{s}$ and expanding the product, it suffices to know for which values of $t+i l$ and $\alpha$ the function $z^{t+i l} v^{\alpha}$ belongs to $\mathcal{H}^{2}\left(\mathbb{H}_{d}\right)$. Let us admit for a while that this is true if and only if $\frac{-d}{2}-\frac{|\alpha|}{2}<t<\frac{d}{2}-\frac{|\alpha|}{2}$. When $t$ moves in this interval and $l$ moves in $\mathbb{R}, \lambda^{t+i l}$ describes the corona

$$
\left\{\frac{\lambda^{-d / 2}}{\lambda^{|\alpha| / 2}}<|z|<\frac{\lambda^{d / 2}}{\lambda^{|\alpha| / 2}}\right\}
$$


which gives the proposition. So, let us study $\left\|z^{t+i l} v^{\alpha}\right\|_{\mathcal{H}^{2}\left(\mathbb{H}_{d}\right)}$ :

$$
\begin{aligned}
\left\|z^{t+i l} v^{\alpha}\right\|_{\mathcal{H}^{2}\left(\mathbb{H}_{d}\right)} & =\int_{\partial \mathbb{H}_{d}} \frac{|z|^{2 t}\left|v^{\alpha}\right|^{2}}{|z+i|^{2 d}} d \sigma_{\partial \mathbb{H}_{d}} \\
& =\int_{\mathbb{R}} \int_{\mathbb{C}^{d-1}} \frac{\left(x^{2}+\|(u, v)\|^{4}\right)^{t} \prod_{i=1}^{s}\left|v_{i}^{\alpha_{i}}\right|^{2}}{\left(x^{2}+\left(1+\|(u, v)\|^{2}\right)^{2}\right)^{d}} d u d v d x
\end{aligned}
$$

where we put $z=x+i\|(u, v)\|^{2}$ in the last integral. We use polar coordinates in the integral depending on $(u, v)$ in $\mathbb{C}^{d-1}$ and we put

$$
(u, v)=r\left(\xi_{2,1}+i \xi_{2,2}, \ldots, \xi_{d-s, 1}+i \xi_{d-s, 2}, \xi_{d-s+1,1}+i \xi_{d-s+1,2}, \ldots, \xi_{d, 1}+i \xi_{d, 2}\right)
$$

with $r \in \mathbb{R}_{+}$and $\xi=\left(\xi_{2,1}, \xi_{2,2}, \ldots, \xi_{d-s, 1}, \xi_{d-s, 2}, \xi_{d-s+1,1}, \xi_{d-s+1,2}, \ldots, \xi_{d, 1}, \xi_{d, 2}\right) \in$ $\mathcal{S}_{2(d-1)-1}$. Next, we can write

$$
\prod_{i=1}^{s}\left|v_{i}^{\alpha_{i}}\right|^{2}=r^{2|\alpha|} \underbrace{\prod_{i=1}^{s}\left|\xi_{d-s+i, 1}+i \xi_{d-s+i, 2}\right|^{2 \alpha_{i}}}_{:=C_{\alpha}(\xi)},
$$

and then

$$
\begin{aligned}
& \|F\|_{\mathcal{H}^{2}\left(\mathbb{H}_{d}\right)}= \\
& \int_{\mathbb{R}} \int_{0}^{+\infty} \int_{\mathcal{S}_{2(d-1)-1}} \frac{\left(x^{2}+r^{4}\right)^{t} r^{2|\alpha|+2(d-1)-1} C_{\alpha}(\xi)}{\left(x^{2}+\left(1+r^{2}\right)^{2}\right)^{d}} d \sigma_{\mathcal{S}_{2(d-1)-1}}(\xi) d r d x= \\
& \quad \int_{\mathbb{R}} \int_{0}^{+\infty} \frac{\left(x^{2}+r^{4}\right)^{t} r^{2|\alpha|+2(d-1)-1}}{\left(x^{2}+\left(1+r^{2}\right)^{2}\right)^{d}} \int_{\mathcal{S}_{2(d-1)-1}} C_{\alpha}(\xi) d \sigma_{\mathcal{S}_{2(d-1)-1}}(\xi) d r d x .
\end{aligned}
$$

As $\mathcal{S}_{2(d-1)-1}$ is bounded and as $\prod_{i=1}^{s}\left|\xi_{d-s+i, 1}+i \xi_{d-s+i, 2}\right|^{2 \alpha_{i}}$ is also bounded and bounded away from 0 on $\mathcal{S}_{2(d-1)-1}$, and using the change of variables $r \longmapsto r^{2}$, we deduce that $F$ is in $\mathcal{H}^{2}\left(\mathbb{H}_{d}\right)$ if and only if

$$
I_{t, \alpha, d}:=\int_{0}^{+\infty} \int_{0}^{+\infty} \frac{\left(x^{2}+r^{2}\right)^{t} r^{|\alpha|+d-2}}{\left(x^{2}+(1+r)^{2}\right)^{d}} d r d x<+\infty .
$$

We shall use the following lemma:

Lemma 3.8. Let $e, f, g \in \mathbb{R}$. The integral

$$
\int_{0}^{1} \int_{0}^{1} x^{e} y^{f}\left(x^{2}+y^{2}\right)^{g} d x d y
$$

is finite if and only if $e+f+2 g>-2$. 
Proof. This is a straightforward computation:

$$
\begin{aligned}
\int_{0}^{1} \int_{0}^{1} x^{e} y^{f}\left(x^{2}+y^{2}\right)^{g} d x d y & <\infty \\
& \Uparrow \text { by the change of variable } y \longmapsto y^{f+1} \\
\int_{0}^{1} x^{e} \int_{0}^{1}\left(x^{2}+y^{\frac{2}{f+1}}\right)^{g} d y d x & <\infty \\
& \Uparrow \\
\int_{0}^{1} x^{e+2 g} \int_{0}^{1}\left(1+\left(\frac{y}{x^{f+1}}\right)^{\frac{2}{f+1}}\right)^{g} d y d x & <\infty \\
& \mathbb{\Downarrow} \text { by the change of variable } y \longmapsto \frac{y}{x^{f+1}} \\
\int_{0}^{1} x^{e+f+2 g+1} \int_{0}^{1}(1+y)^{g} d y d x & <\infty,
\end{aligned}
$$

which is equivalent to $e+f+2 g+1>-1$ as we wished.

We come back to the study of the integrability of $I_{t, \alpha, d}$. On the compact set $[0,1] \times[0,1]$, since $\left(x^{2}+(1+r)^{2}\right)$ is greater than 1 , we just have to study the integrability of $(r, x) \longmapsto r^{d+|\alpha|-2}\left(x^{2}+r^{2}\right)^{t}$. This follows directly from the lemma, and the integrability on $[0,1] \times[0,1]$ is equivalent to the condition $t>-\frac{d}{2}-\frac{|\alpha|}{2}$.

On $[1,+\infty] \times[1,+\infty]$, we are reduced to studying the integrability of

$$
(r, x) \longmapsto r^{d+|\alpha|-2}\left(x^{2}+r^{2}\right)^{t-d} .
$$

Using the changes of variables $x \longmapsto \frac{1}{x}$ and $r \longmapsto \frac{1}{r}$, we come back to the situation of Lemma 3.8 for the function

$$
(x, r) \longmapsto r^{d-|\alpha|-2 t} x^{-2 t+2 d-2}\left(x^{2}+r^{2}\right)^{t-d} .
$$

After a small computation, we find that $I_{t, \alpha, d}$ is finite if and only if $t<\frac{d}{2}-\frac{|\alpha|}{2}$, which was the missing inequality.

3.6. Examples. Let $\psi_{1}(z, w)=(4 z, w)$ and $\psi_{2}(z, w)=(4 z, w / 10)$, acting on $\mathbb{H}_{2}$. We know that

$$
\sigma\left(C_{\psi_{1}}\right)=\bigcup_{n \geq 0}\left\{\frac{1}{4}\left(\frac{1}{2}\right)^{n} \leq|z| \leq 4\left(\frac{1}{2}\right)^{n}\right\} \cup\{0\}:=\bigcup_{n \geq 0} C_{n} \cup\{0\} .
$$

Now, the coronae $C_{n}$ and $C_{n+1}$ do intersect, and apparently the spectrum of $C_{\psi_{1}}$ does not look like a union of coronae: it is the disk $\bar{D}(0,4)$. This phenomenon does not occur for $C_{\psi_{2}}$, because in that case the coronae are disjoint.

Thus, even when $\phi$ acts as an automorphism on a slice, its spectrum can be a disk. However, it is better to think that it is a union of coronae which intersect rather than to think that it is a disk. This point of view will be crucial for the study of the dynamics of the associated composition operator. 


\section{DyNAMiCS OF HYPERBOLIC LINEAR FRACTIONAL COMPOSITION OPERATORS}

4.1. Introduction. The study of their dynamics is an important aspect of the theory of composition operators (see for instance the books [6, [15]). Regarding linear fractional maps of the ball, several partial results have been obtained (in 9] for the automorphism case, in [16] for a special case of hyperbolic maps) and a complete study of the parabolic case has been done in 2,1$]$ We shall completely solve here the study of the hyperbolic case, closing the subject at least for composition operators on the Hardy space $H^{2}\left(\mathbb{B}_{d}\right)$. The result that we will prove is more precise than the announced Theorem 1.5. To state it, we need a definition:

Definition 4.1. Let $X$ be a separable complex Banach space and let $T \in \mathcal{L}(X)$. $T$ is called chaotic if $T$ is hypercyclic and has a dense set of periodic points.

In other words, $T$ is chaotic if and only if it is hypercyclic and

$$
\operatorname{span}\left(\operatorname{ker}(T-z), z \in e^{2 \pi i \mathbb{Q}}\right)
$$

is dense in $X$.

Let us now give the complete statement that we shall prove. Observe that the dynamical properties of $C_{\phi}$ are uniquely determined by the signature of $\phi$.

Theorem 4.2. Let $\phi \in L F M\left(\mathbb{B}_{d}\right)$ be hyperbolic and univalent.

(1) If $p_{\phi}=d-1$, then

(a) $\mu C_{\phi}$ is chaotic when $\varepsilon_{\phi}=1$ and $\mu$ belongs to the corona $\left\{\lambda^{-d / 2}<\right.$ $\left.|\mu|<\lambda^{d / 2}\right\}$

(b) $\mu C_{\phi}$ is chaotic when $\varepsilon_{\phi}=0$ and $\mu$ belongs to $\mathbb{C} \backslash \overline{D\left(0, \lambda^{-d / 2}\right)}$.

(2) If $p_{\phi}<d-1$, then

(a) $C_{\phi}$ is never hypercyclic;

(b) $C_{\phi}$ is supercyclic iff $\varepsilon_{\phi}=0$;

(c) $C_{\phi}$ is always cyclic.

We will first prove part (1) of the above theorem. Following a method of M. Taniguchi [22, we will not prove directly that $\operatorname{span}\left(\operatorname{ker}(T-z), z \in e^{2 \pi i \mathbb{Q}}\right)$ is dense. We will rather apply an indirect method based on the following chaoticity criterion (see [3, Theorem 6.10]).

Theorem 4.3 (Chaoticity criterion). Let $X$ be a separable complex Banach space and let $T \in \mathcal{L}(X)$. Assume that there exist a dense set $\mathcal{D} \subset X$ and a sequence of maps $S_{n}: \mathcal{D} \rightarrow \mathcal{D}$ such that

(1) $\sum T^{n}(x)$ and $\sum S_{n}(x)$ are unconditionnaly convergent, for each $x \in \mathcal{D}$;

(2) $T^{n} S_{n}=I$ on $\mathcal{D}$ for each $n$.

Then $T$ is chaotic.

The most difficult part of Theorem 4.2 is the proof of $(2)(\mathrm{b})$. The basic tool will be the outer supercyclicity criterion given by N. Feldman, V. Miller and T. Miller in [14]:

Theorem 4.4 (Outer supercyclicity criterion). Let $X$ be a Banach space and let $T \in \mathcal{L}(X)$. Suppose that there exists a dense linear subspace $Y$ and that, for every

\footnotetext{
${ }^{1}$ A result for hyperbolic linear fractional maps was announced in [5]. Unfortunately, there is a mistake in the proof and the result which was announced is not correct.
} 
$y \in Y$, there exists a dense linear subspace $X_{y}$ such that:

(1) there exist functions $S_{n}: Y \rightarrow X$ such that $T^{n} S_{n} y=y$ for all $y \in Y$, and

(2) for any $y \in Y$ and any $x \in X_{y}$, then $\left\|T^{n} x\right\| \cdot\left\|S_{n} y\right\| \rightarrow 0$ as $n \rightarrow \infty$.

Then $T$ is supercyclic.

The main difficulty consists in exhibiting the subspaces $Y$ and $X_{y}$. At this point, in a surprising fashion, the result of part (1) will come into play. Indeed, with our method, we obtain a posteriori that, under the assumptions of part (1)(b) of Theorem 4.2, the subspace span $\left(\operatorname{ker}\left(\mu C_{\phi}-\lambda\right), \lambda \in e^{2 \pi i \mathbb{Q}}\right)$ is dense. Now, it turns out that these vectors also have a good behavior under the action of $C_{\phi}$, even when $\phi$ satisfies the assumptions of part (2)(b). This will allow the construction of $Y$ and $X_{y}$.

Finally, the result concerning cyclicity will be a consequence of the following theorem (see [2, Corollary 6.3]):

Theorem 4.5. Let $T \in \mathcal{L}(X)$ be such that, for any $\mu \in \mathbb{C}, \bigcup_{P \in \mathcal{P}_{\mu}} \operatorname{ker} P(T)$ is dense, where $\mathcal{P}_{\mu}=\{P \in \mathbb{C}[z], P(\mu) \neq 0\}$. Then $T$ is cyclic.

4.2. Proof of chaoticity. In this section, we will prove part (1) of Theorem 4.2 and we will give some interesting consequences of it. We begin with a technical lemma.

Lemma 4.6. Let $a \in \mathbb{R}_{+}$. The following estimates hold true:

$$
I:=\int_{\substack{(z, w) \in \partial \mathbb{H}_{d} \\|z| \geq a}} \frac{d \sigma}{|z+i|^{2 d}} \lesssim \frac{1}{a^{d}} \text { and } \int_{\substack{(z, w) \in \partial \mathbb{H}_{d} \\|z| \leq a}} \frac{d \sigma}{|z+i|^{2 d}} \lesssim a^{d} .
$$

Proof. The proof consists in a straightforward computation. Successively passing to polar coordinates in $I$, making the change of variable $y=r^{2}$, then putting $u=y^{d-1}$ and $v=\frac{u}{x^{d-1}}$, we get

$$
\begin{aligned}
I & \leq \int_{0}^{+\infty} \int_{\max \left(0,(a-x)^{1 / 2}\right)}^{+\infty} \frac{r^{2 d-3}}{\left(x^{2}+r^{4}\right)^{d}} d r d x \\
& \leq \int_{0}^{+\infty} \int_{\max (0,(a-x))}^{+\infty} \frac{y^{d-2}}{\left(x^{2}+y^{2}\right)^{d}} d y d x \\
& \lesssim \int_{0}^{+\infty} \int_{\max \left(0,(a-x)^{d-1}\right)}^{+\infty} \frac{d u}{x^{2 d}\left(1+\left(\frac{u}{x^{d-1}}\right)^{2 /(d-1)}\right)^{d}} d x \\
& \lesssim \int_{0}^{+\infty} \int_{\max \left(0,\left(\frac{a-x}{x}\right)^{d-1}\right)}^{+\infty} \frac{d v}{x^{d+1}\left(1+v^{2 /(d-1)}\right)^{d}} d x .
\end{aligned}
$$

Now we cut the last integral into two parts. First on the part $x \geq a / 2$, we write

$$
\int_{a / 2}^{+\infty} \int_{0}^{+\infty} \frac{d v}{x^{d+1}\left(1+v^{2 /(d-1)}\right)^{d}} d x=\int_{a / 2}^{+\infty} \frac{d x}{x^{d+1}} \int_{0}^{+\infty} \frac{d v}{\left(1+v^{2 /(d-1)}\right)^{d}} \lesssim \frac{1}{a^{d}} .
$$

Next, on the part $0 \leq x \leq a / 2$, using

$$
\int_{c}^{+\infty} \frac{1}{\left(1+x^{\alpha}\right)^{\beta}} d x \lesssim \min \left(1, \frac{1}{c^{\alpha \beta-1}}\right)
$$


we get

$$
\begin{aligned}
\int_{0}^{a / 2} \int_{\left(\frac{a-x}{x}\right)^{d-1}}^{+\infty} \frac{d v}{x^{d+1}\left(1+v^{2 /(d-1)}\right)^{d}} d x & \lesssim \int_{0}^{a / 2} \frac{x^{\left(\frac{2 d}{d-1}-1\right)(d-1)}}{x^{d+1}(a-x)^{\left(\frac{2 d}{d-1}-1\right)(d-1)} d x} \\
& \lesssim \int_{0}^{a / 2} \frac{d x}{(a-x)^{d+1}} \\
& \lesssim \frac{1}{a^{d}}
\end{aligned}
$$

The proof of the second estimate is easier and just follows from a volume argument.

Let us start now with a normal form of $\phi$ which may be written as

$$
\psi(z, w)=(\lambda z+b, D w)
$$

with $\lambda^{-1 / 2} D$ unitary, $\Im m(b) \geq 0$. We need to study the chaoticity of $\mu C_{\psi}$ acting on $\mathcal{H}^{2}\left(\mathbb{H}_{d}\right)$. The main idea of the proof is that $\psi$ induces an automorphism of its characteristic domain, with an attractive fixed point, equal to $\infty$, for $\psi$ and an attractive fixed point, denoted by $z_{0}$, for $\psi^{-1}$. Choosing for $\mathcal{D}$ functions vanishing both in $\infty$ and in $z_{0}$, we will be able to apply Theorem 4.3 .

Let us proceed with the details. Let $\alpha=1$ and $\beta=\left(z_{0}-i\right) /\left(z_{0}+i\right)$ with $z_{0}=-b /(\lambda-1)$. Notice that $\beta \notin \mathbb{D}$ since $\Im m(b) \geq 0$. For $k \geq 0$, we define the subspace $\mathcal{D}_{0, k}$ of $H^{2}\left(\mathbb{B}_{d}\right)$ as follows:

$$
\mathcal{D}_{0, k}:=\left\{(z-\alpha)^{k}(z-\beta)^{k} Q(z, w), Q \text { polynomial in }(z, w) \in \mathbb{C} \times \mathbb{C}^{d-1}\right\} .
$$

Since $\alpha, \beta \notin \mathbb{D}$, using the series representation of functions in $H^{2}\left(\mathbb{B}_{d}\right)$ and the Hilbertian properties of this space, it is easy to prove that $\mathcal{D}_{0, k}$ is dense in $H^{2}\left(\mathbb{B}_{d}\right)$. Denoting by $\mathcal{D}_{k}$ the analogue of $\mathcal{D}_{0, k}$ in the upper half-space,

$$
\mathcal{D}_{k}=\left\{P \circ \sigma_{c}^{-1}, P \in \mathcal{D}_{0, k}\right\},
$$

where $\sigma_{c}: \mathbb{B}_{d} \rightarrow \mathbb{H}_{d}$ is the Cayley map, it remains true that $\mathcal{D}_{k}$ is dense in $\mathcal{H}^{2}\left(\mathbb{H}_{d}\right)$ and it is sufficient to find some $k$ (large enough...) such that (1) and (2) of Theorem 4.3 are verified for $T=\mu C_{\psi}$ and for some convenient operator $S$.

We need another lemma.

Lemma 4.7. Let $\Omega$ be the characteristic domain of $\psi$. Then $\sigma_{c}^{-1}(\Omega)$ is bounded.

Proof. Recall that

$$
\Omega=\left\{(z, w) \in \mathbb{C}^{d}, \Im m(z)>\|w\|^{2}-\frac{\Im m(b)}{\lambda-1}\right\}
$$

and that

$$
\sigma_{c}^{-1}(z, w)=\left(\frac{z-i}{z+i}, \frac{2 w}{z+i}\right) .
$$

First, $(z, w) \mapsto(z-i) /(z+i)$ is bounded on $\Omega$ because

$$
\left|\frac{z-i}{z+i}\right|^{2}=\frac{\Re e(z)^{2}+(\Im m(z)-1)^{2}}{\Re e(z)^{2}+(\Im m(z)+1)^{2}}
$$

and

$$
\Im m(z)+1 \geq 1-\frac{\Im m(b)}{\lambda-1}>0
$$


This is the place where we need to impose, in the normal form, the condition $\Im m(b) \in(0, \lambda-1)$. Next, $(z, w) \mapsto 2 w /(z+i)$ is also bounded on $\Omega$ since

$$
\left\|\frac{2 w}{z+i}\right\|^{2}=\frac{2\|w\|^{2}}{\Re e(z)^{2}+(\Im m(z)+1)^{2}} \leq \frac{2 \Im m(z)}{\Re e(z)^{2}+(\Im m(z)+1)^{2}}
$$

and we conclude the proof using (5) again.

The previous lemma has the following interesting consequence. Let $R \in \mathcal{D}_{k}$. There is some polynomial $Q(z, w)$ such that

$$
R(z, w)=\left[(z-\alpha)^{k}(z-\beta)^{k} Q(z, w)\right] \circ \sigma_{c}^{-1} .
$$

Because of Lemma 4.7, there exist $M, C_{1}, C_{2} \in \mathbb{R}$ such that $Q,(z-\alpha)^{k}$ and $(z-\beta)^{k}$ are respectively bounded by $M, C_{1}$ and $C_{2}$ on $\sigma_{c}^{-1}(\Omega)$. Then, for every $(z, w) \in \Omega, R(z, w)$ is well-defined and we get the following estimations:

$$
\begin{aligned}
|R(z, w)| & \leq C_{2} M\left|\frac{z-i}{z+i}\right|^{k} \leq \frac{C}{|z+i|^{k}}, \\
|R(z, w)| & \leq C_{1} M\left|\frac{z-i}{z+i}-\frac{z_{0}-i}{z_{0}+i}\right|^{k} \leq C\left|z-z_{0}\right|^{k} .
\end{aligned}
$$

Let $\mathcal{C}=\mathbb{C} \backslash \overline{D\left(0, \lambda^{-d / 2}\right)}$ if $\Im m(b)>0$ and $\mathcal{C}=\left\{\lambda^{-d / 2}<|\mu|<\lambda^{d / 2}\right\}$ when $\Im m(b)=$ 0 . For $\mu \in \mathcal{C}, n \geq 1$ and $R \in \mathcal{D}_{k}$, we define

$$
\begin{aligned}
S_{n}(R) & =\mu^{-n} R\left(\lambda^{-n} z-\frac{\lambda^{-n}-1}{\lambda^{-1}-1} \lambda b, D^{-n} w\right) \\
& =\mu^{-n} R \circ \psi_{n}^{-1}(z, w) .
\end{aligned}
$$

This is a well-defined formula since, for any $(z, w) \in \mathbb{H}_{d}, \psi_{n}^{-1}(z, w) \in \Omega$. Moreover, $\left(\mu C_{\psi}\right)^{n} \circ S_{n}=I$. Therefore, it remains to show that condition (1) of Theorem 4.3 is satisfied.

Step 1. $\sum_{n \geq 0}\left\|S_{n}(R)\right\|<+\infty$ when $k$ is large enough.

Let $\vartheta \in(0,1)$ such that $|\mu|^{2}>\lambda^{-\vartheta d}$. We cut the integral into two parts:

$$
\begin{aligned}
\left\|S_{n}(R)\right\|_{\mathcal{H}^{2}}^{2} \leq|\mu|^{-2 n} \int_{\substack{(z, w) \in \partial \mathbb{H}_{d} \\
|z| \leq \lambda^{\vartheta n}}} \frac{\left|R\left(\lambda^{-n} z-\frac{\lambda^{-n}-1}{\lambda^{-1}-1} \lambda b, D^{-n} w\right)\right|^{2}}{|z+i|^{2 d}} d \sigma_{\partial \mathbb{H}_{d}} \\
+|\mu|^{-2 n} \int_{\substack{(z, w) \in \partial \mathbb{H}_{d} \\
|z| \geq \lambda^{\vartheta n}}} \frac{\left|R\left(\lambda^{-n} z-\frac{\lambda^{-n}-1}{\lambda^{-1}-1} \lambda b, D^{-n} w\right)\right|^{2}}{|z+i|^{2 d}} d \sigma_{\partial \mathbb{H}_{d}} .
\end{aligned}
$$

First, since $R$ is bounded on $\Omega$, Lemma 4.6 implies that

$$
|\mu|^{-2 n} \int_{\substack{(z, w) \in \partial \mathbb{H}_{d} \\|z| \geq \lambda^{\vartheta n}}} \frac{\left|R\left(\lambda^{-n} z-\frac{\lambda^{-n}-1}{\lambda^{-1}-1} \lambda b, D^{-n} w\right)\right|^{2}}{|z+i|^{2 d}} d \sigma_{\partial \mathbb{H}_{d}} \lesssim\left(\frac{|\mu|^{-2}}{\lambda^{\vartheta d}}\right)^{n} .
$$


Next, the inequality (7) above yields

$$
\begin{aligned}
& |\mu|^{-2 n} \int_{\substack{(z, w) \in \partial \mathbb{H}_{d} \\
|z| \leq \lambda^{\vartheta n}}} \frac{\left|R\left(\lambda^{-n} z-\frac{\lambda^{-n}-1}{\lambda^{-1}-1} \lambda b, D^{-n} w\right)\right|^{2}}{|z+i|^{2 d}} d \sigma_{\partial \mathbb{H}_{d}} \\
& \quad \lesssim|\mu|^{-2 n} \int_{\substack{(z, w) \in \partial \mathbb{H}_{d} \\
|z| \leq \lambda^{\vartheta n}}}\left|\lambda^{-n} z-\frac{\lambda^{-n}-1}{\lambda^{-1}-1} \lambda b-z_{0}\right|^{2 k} d \sigma_{\partial \mathbb{H}_{d}} \\
& \quad \lesssim|\mu|^{-2 n} \int_{\substack{(z, w) \in \partial \mathbb{H}_{d} \\
|z| \leq \lambda^{\vartheta n}}}\left|\lambda^{-n}\left(z-z_{0}\right)\right|^{2 k} d \sigma_{\partial \mathbb{H}_{d}} \\
& \lesssim \frac{|\mu|^{-2 n}}{\lambda^{2(1-\vartheta) n k}} .
\end{aligned}
$$

Thus, $\sum_{n \geq 0}\left\|S_{n}(R)\right\|$ is convergent, assuming $|\mu|>\lambda^{-d / 2}$ and $k$ is large enough.

Step 2. $\sum_{n \geq 0}\left\|\left(\mu C_{\psi}\right)^{n}(R)\right\|<+\infty$ when $k$ is large enough.

For this step, we have to distinguish the cases $\Im m(b)=0$ and $\Im m(b)>0$. When $\Im m(b)>0$, observe that for any $(z, w) \in \partial \mathbb{H}_{d}$,

$$
\left|\lambda^{n} z+\frac{\lambda^{n}-1}{\lambda-1} b+i\right| \geq \Im m\left(\frac{\lambda^{n}-1}{\lambda-1} b\right) \gtrsim \lambda^{n} .
$$

This yields

$$
\begin{aligned}
\left\|\left(\mu C_{\psi}\right)^{n}(R)\right\|_{\mathcal{H}^{2}}^{2} & =|\mu|^{2 n} \int_{\partial \mathbb{H}_{d}} \frac{\left|R\left(\lambda^{n} z+\frac{\lambda^{n}-1}{\lambda-1} b, D^{n} w\right)\right|^{2}}{|z+i|^{2 d}} d \sigma_{\partial \mathbb{H}_{d}} \\
& \lesssim\left(\frac{|\mu|}{\lambda^{k}}\right)^{2 n}
\end{aligned}
$$

by inequality (6) above. Thus, for $k$ large enough, $\sum_{n \geq 0}\left\|\left(\mu C_{\psi}\right)^{n}(R)\right\|_{\mathcal{H}^{2}}$ is convergent, and this property does not depend on the value of $|\mu|$.

When $\Im m(b)=0$ (in fact, $b=0$ by our simplifications), we proceed as in Step 1 , splitting the integral into two parts:

$$
\begin{aligned}
\left\|C_{\psi}^{n}(R)\right\|_{\mathcal{H}^{2}}= & |\mu|^{2 n} \int_{\substack{(z, w) \in \partial \mathbb{H}_{d} \\
|z| \leq \lambda^{-\vartheta n}}}\left|R\left(\lambda^{n} z, D^{n} w\right)\right|^{2} d \sigma_{\partial \mathbb{H}_{d}} \\
& +|\mu|^{2 n} \int_{\substack{(z, w) \in \mathbb{H}_{d} \\
|z| \geq \lambda^{-\vartheta n}}}\left|R\left(\lambda^{n} z, D^{n} w\right)\right|^{2} d \sigma_{\partial \mathbb{H}_{d}},
\end{aligned}
$$

where $\vartheta \in(0,1)$ is such that $|\mu|^{2}<\lambda^{\vartheta d}$. For the second integral, we may use inequality (6) satisfied by $R$ above and fix $k$ large enough so that this part becomes the term of a convergent series. For the first integral, we appeal to (the easy part of) Lemma 4.6 to conclude the proof (this is the point where the condition $|\mu|^{2}<\lambda^{d}$ comes into play).

Application. We conclude this subsection by an application to the density of eigenvectors. Suppose that $\Im m(b)>0$ and let $r>\lambda^{-d / 2}$. Also let $\psi_{0}(z, w)=$ $(\lambda z+b, \sqrt{\lambda} w)$. Since $r C_{\psi_{0}}$ is chaotic, we know that

$$
\operatorname{span}\left(\operatorname{ker}\left(r C_{\psi_{0}}-e^{i \theta}\right), \theta \in \mathbb{R}\right) \text { is dense. }
$$


Let us introduce a decomposition of $\mathcal{H}^{2}\left(\mathbb{H}_{d}\right)$ slightly different from that of Section 3. For $\alpha \in \mathbb{N}^{d-1}$, we denote by $\mathcal{E}_{\alpha}$ the set of functions $f$ of $\mathcal{H}^{2}\left(\mathbb{H}_{d}\right)$ which may be written in the form $f(z, w)=F(z) w^{\alpha}$, so that we can decompose $\mathcal{H}^{2}\left(\mathbb{H}_{d}\right)$ as $\bigoplus_{\alpha \in \mathbb{N}^{d-1}} \mathcal{E}_{\alpha}$. Each $\mathcal{E}_{\alpha}$ is stable under $C_{\psi_{0}}$, so that

$$
\operatorname{span}\left(\operatorname{ker}\left(r C_{\psi_{0} \mid \mathcal{E}_{\alpha}}-e^{i \theta}\right), \theta \in \mathbb{R}\right) \text { is dense in } \mathcal{E}_{\alpha} .
$$

Now, for $f(z, w)=F(z) w^{\alpha}$, one has

$$
r C_{\psi_{0}} f=e^{i \theta} f \Longleftrightarrow F(\lambda z+b)=\frac{\lambda^{-|\alpha| / 2} e^{i \theta}}{r} F(z) .
$$

This means that we have obtained the following corollary:

Corollary 4.8. For each $\alpha \in \mathbb{N}^{d-1}$, for each $r>\lambda^{-d / 2}$, for each $b \in \mathbb{C}$ with $\Im m(b)>0$,

$$
\mathcal{E}_{\alpha, r, b}:=\operatorname{span}\left(F(z) w^{\alpha} \in \mathcal{H}^{2}\left(\mathbb{H}_{d}\right) ; \exists \theta \in \mathbb{R}, F(\lambda z+b)=\frac{\lambda^{-|\alpha| / 2} e^{i \theta}}{r} F(z)\right)
$$

is dense in $\mathcal{E}_{\alpha}$.

If we now start with $\psi_{0}(z)=(\lambda z, \sqrt{\lambda} w)$, then we get

Corollary 4.9. For each $\alpha \in \mathbb{N}^{d-1}$, for each $r \in\left(\lambda^{-d / 2}, \lambda^{d / 2}\right)$,

$$
\mathcal{E}_{\alpha, r, 0}:=\operatorname{span}\left(F(z) w^{\alpha} \in \mathcal{H}^{2}\left(\mathbb{H}_{d}\right) ; \exists \theta \in \mathbb{R}, F(\lambda z)=\frac{\lambda^{-|\alpha| / 2} e^{i \theta}}{r} F(z)\right)
$$

is dense in $\mathcal{E}_{\alpha}$.

4.3. Proof of non-hypercyclicity. We suppose that $\psi \in L F M\left(\mathbb{H}_{d}\right)$ is given by

$$
\psi(z, u, v)=(\lambda z+b, D u, A v+c)
$$

with $\operatorname{dim} v>0$ and we have to prove that $C_{\psi}$ is not hypercyclic. This is the easy part of the proof which depends on the following lemma. The notation is that of Section 3 .

Lemma 4.10. There exists $N \in \mathbb{N}$ such that, if we denote by $P_{N}$ the orthogonal projection on $\mathcal{K}_{N}$, then $\left\|P_{N} \circ C_{\psi \mid \mathcal{K}_{N}}\right\|<1$.

Proof. The proof goes along the same lines as that of Lemma 3.5. Recall that $C_{\psi}$ is upper-triangular in the decomposition $\mathcal{H}^{2}\left(\mathbb{H}_{d}\right)=\bigoplus_{|\alpha|<N} \mathcal{H}_{\alpha} \oplus \mathcal{K}_{N}$. Let $A=U \Sigma V$ be the singular value decomposition of $A$. We factorize $C_{\psi}$ as $C_{\psi}=C_{\tau_{1}} \circ C_{\psi_{\Sigma}} \circ C_{\tau_{2}}=$ $C_{\tau_{2} \circ \psi_{\Sigma} \circ \tau_{1}}$, where

$$
\left\{\begin{aligned}
\tau_{1}:(z, w) & \mapsto(z, u, V v) \\
\psi_{\Sigma}:(z, w) & \mapsto\left(\lambda z+b, D u, \Sigma v+U^{-1} c\right), \\
\tau_{2}:(z, w) & \mapsto(z, u, U v) .
\end{aligned}\right.
$$

Since $C_{\tau_{1}}$ and $C_{\tau_{2}}$ are isometries of $\mathcal{H}^{2}\left(\mathbb{H}_{d}\right)$ and since they both preserve $\mathcal{K}_{N}$ and $\mathcal{K}_{N}^{\perp}$, it suffices to establish $\left\|P_{N} \circ C_{\psi_{\Sigma} \mid \mathcal{K}_{N}}\right\|<1$ for $N$ large enough. Let us finally introduce $\psi_{0}(z, w)=(\lambda z+b, D u, \Sigma v)$. Clearly, $P_{N} \circ C_{\psi_{\Sigma} \mid \mathcal{K}_{N}}=C_{\psi_{0} \mid \mathcal{K}_{N}}$. We can now conclude because, as in Lemma 3.5, the sequence $\left(\left\|C_{\psi_{0} \mid \mathcal{K}_{N}}\right\|\right)$ goes to 0 as $N$ goes to infinity. 
Let us now deduce that $C_{\psi}$ is not hypercyclic. Suppose that the contrary holds and let $f=f_{1}+f_{2}$ be a hypercyclic vector with $f_{1} \in \mathcal{K}_{N}^{\perp}$ and $f_{2} \in \mathcal{K}_{N}$, with $N \geq 0$. The set $\left\{P_{N} C_{\psi}^{n} f: n \geq 0\right\}$ has to be dense in $\mathcal{K}_{N}$. Since $C_{\psi}$ is uppertriangular in the decomposition $\mathcal{K}_{N}^{\perp} \oplus \mathcal{K}_{N}$, this means that $\left\{\left(P_{N} \circ C_{\psi}\right)^{n} f_{2}: n \geq 0\right\}$ has to be dense. When $N$ is large enough, this is impossible, since the sequence $\left(\left(P_{N} \circ C_{\psi}\right)^{n} f_{2}\right)_{n \geq 0}$ goes to zero.

4.4. Proof of supercyclicity. We suppose now that $\psi$ is given by the formula

$$
\psi(z, u, v)=(\lambda z+b, D u, A v+c)
$$

with $\Im m(b)>0$ and we intend to show that $C_{\psi}$ is supercyclic. We will apply the outer supercyclicity criterion with $Y=\bigoplus_{\alpha \in \mathbb{N}^{d-1}} \mathcal{E}_{\alpha, r, b}$ for some fixed $r>\lambda^{-d / 2}$. $Y$ is dense in $\mathcal{H}^{2}\left(\mathbb{H}_{d}\right)$ by Corollary 4.8 .

By linearity it suffices, for each $y=F(z) w^{\alpha} \in \mathcal{E}_{\alpha, r, b}$, with $F(\lambda z+b)=$ $r^{-1} \lambda^{-|\alpha| / 2} e^{i \theta} F(z)$ and $\alpha \in \mathbb{N}^{d-1}$, to find a subset $X_{y} \subset \mathcal{H}^{2}\left(\mathbb{H}_{d}\right)$ and a sequence of maps $\left(S_{n}\right)$ satisfying the assumptions of the outer supercyclicity criterion. The idea is that, although $C_{\psi}$ is not invertible, it will be invertible on a finite-dimensional space containing $y$, allowing the construction of the sequence $\left(S_{n} y\right)$.

From now on, we will rather write

$$
\psi(z, w)=(\lambda z+b, M w+c),
$$

where $M=\left[\begin{array}{ll}D & 0 \\ 0 & A\end{array}\right]$ is an upper triangular matrix. Also let $N=|\alpha|$ and let us consider the following finite-dimensional subspace of $\mathcal{H}^{2}\left(\mathbb{H}_{d}\right)$ :

$$
Z=\left\{\sum_{|\beta| \leq N} a_{\beta} F(z) w^{\beta}: a_{\beta} \in \mathbb{C}\right\} .
$$

$Z$ is contained in $\mathcal{H}^{2}\left(\mathbb{H}_{d}\right)$, since $F(z) w^{\beta}$ belongs to $\mathcal{H}^{2}\left(\mathbb{H}_{d}\right)$ as soon as $F(z) w^{\alpha}$ belongs to $\mathcal{H}^{2}\left(\mathbb{H}_{d}\right)$, for any $|\beta| \leq|\alpha|$. We endow $\mathbb{N}^{d-1}$ with the order defined in Section 3. Hence, we get immediately that $Z$ is mapped into itself by $C_{\psi}$ and that the matrix of $C_{\psi \mid Z}$ with respect to the canonical basis $\left(F(z) w^{\beta}\right)_{|\beta| \leq N}$ is uppertriangular with non-zero coefficients on the diagonal. This implies that the map $C_{\psi \mid Z}: Z \rightarrow Z$ is invertible. In other words, there exists a constant $C$ (which depends on $y$ ) such that, for any $f \in Z$, we may find $g \in Z$ with $C_{\psi}(g)=f$ and $\|g\| \leq C\|f\|$. It is now easy to construct by induction a sequence $\left(S_{n} y\right)$ satisfying $\left\|S_{n} y\right\| \leq C^{n}\|y\|$ and $C_{\psi}^{n} S_{n} y=y$.

Let us now proceed with the construction of $X_{y}$. Let us consider $\gamma \in \mathbb{N}^{d-1}$. By linearity, it is sufficient to construct a dense subspace $X_{y, \gamma}$ of $\mathcal{E}_{\gamma}$ such that, for any $x \in X_{y, \gamma},\left\|C_{\psi}^{n} x\right\| \cdot\left\|S_{n} y\right\|$ goes to zero. We shall prove that $\mathcal{E}_{\gamma, \rho, b}$, for a sufficiently large $\rho$, is convenient. By linearity again, it is sufficient to deal with any $x=G(z) w^{\gamma}$ with $G(\lambda z+b)=\rho^{-1} \lambda^{-|\alpha| / 2} e^{i \delta} G(z)$. We point out that the condition we will impose on $\rho$ must be independent of $x$ (nevertheless, it can depend on $\gamma$ ).

Let $Z_{0}:=\left\{\sum_{|\beta| \leq|\gamma|} a_{\beta} w^{\beta}: a_{\beta} \in \mathbb{C}\right\}$, endowed with the $\ell^{2}$-norm:

$$
\left\|\sum_{|\beta| \leq|\gamma|} a_{\beta} w^{\beta}\right\|^{2}=\sum_{|\beta| \leq|\gamma|}\left|a_{\beta}\right|^{2} .
$$


Also let $T$ be the self-map of $Z_{0}$ defined by $T\left(w^{\beta}\right)=(M w+c)^{\beta}$. $T$ defines a bounded operator on the finite-dimensional space $Z_{0}$, and $\|T\|$ just depends on $|\gamma|$. Furthermore,

$$
\begin{aligned}
\left\|C_{\psi}^{n}(x)\right\|^{2} & =\frac{\lambda^{-|\alpha| n}}{\rho^{2 n}} \int_{\partial \mathbb{H}_{d}} \frac{|G(z)|^{2}\left|T^{n}\left(w^{\gamma}\right)\right|^{2}}{|z+i|^{2 d}} d \sigma \\
& =\frac{\lambda^{-|\alpha| n}}{\rho^{2 n}} \int_{\partial \mathbb{H}_{d}} \frac{|G(z)|^{2} \sum_{\beta}\left|a_{\beta}\right|^{2}\left|w^{\beta}\right|^{2}}{|z+i|^{2 d}} d \sigma
\end{aligned}
$$

where $\left(a_{\beta}\right)_{|\beta| \leq|\gamma|}$ is defined by $T^{n}\left(w^{\gamma}\right)=\sum_{\beta} a_{\beta} w^{\beta}$. In particular, $\sum\left|a_{\beta}\right|^{2} \leq\|T\|^{2 n}$, so that there exists a constant $D$ (which depends on $x$ ) such that

$$
\left\|C_{\psi}^{n}(x)\right\| \leq D \frac{\|T\|^{n} \lambda^{-|\alpha| n / 2}}{\rho^{n}} .
$$

This yields

$$
\left\|S_{n} y\right\| \cdot\left\|C_{\psi}^{n}(x)\right\| \leq D \frac{C^{n}\|T\|^{n} \lambda^{-|\alpha| n / 2}}{\rho^{n}} .
$$

It suffices to choose $\rho>C\|T\| \lambda^{-|\alpha| / 2}$ (and this choice is independent of $x$ ) to end the proof of the supercyclic part of Theorem 4.2

4.5. Proof of cyclicity and non-supercyclicity. In this last subsection, we work with $\psi \in \operatorname{LFM}\left(\mathbb{H}_{d}\right)$ univalent, which may be written as

$$
\psi(z, u, v)=(\lambda z, D u, A v)
$$

with $\operatorname{dim}(v)>0$, and we have to prove that $C_{\psi}$ is cyclic and not supercyclic.

We prove the non-supercyclicity of $C_{\psi}$ by spectral considerations. Indeed, let $H_{0}=\mathcal{H}_{0}, H_{1}=\bigoplus_{1<|\alpha|<N} \mathcal{H}_{\alpha}$ and $H_{2}=\mathcal{K}_{N}$ for some large $N . C_{\psi}$ is diagonal with respect to the decomposition $\mathcal{H}^{2}\left(\mathbb{H}_{d}\right)=H_{0} \oplus H_{1} \oplus H_{2}$. Moreover, the work of Section 3 shows, at least if $N$ is large enough, that there exists $R_{0}>R_{2}$ such that, for any $f_{0} \in H_{0}$ and any $f_{2} \in H_{2}$,

$$
\left\|C_{\psi}^{n} f_{0}\right\| \geq R_{0}^{n}\left\|f_{0}\right\| \text { and }\left\|C_{\psi}^{n} f_{2}\right\| \leq R_{2}^{n}\left\|f_{2}\right\|
$$

That $C_{\psi}$ is non-supercyclic follows now from a standard argument in linear dynamics, similar to that which proves that each component of the spectrum of a supercyclic operator has to intersect some circle (see [3, Theorem 1.24]).

To prove that $C_{\psi}$ is cyclic, it is convenient to write it as

$$
\psi(z, w)=(\lambda z, M w) .
$$

We fix $\mu \in \mathbb{C}^{d}, \alpha \in \mathbb{N}^{d-1}$ and we observe that $M$ defines, by composition, a linear map $L$ on the finite-dimensional space of homogeneous polynomials of degree $|\alpha|$. Moreover, since $M$ is invertible, it is easy to prove that $L$ is also invertible (for instance, since $M$ is upper-triangular, $L$ is also upper-triangular in the basis $\left(w^{\beta}\right)_{|\beta|=|\alpha|}$ ordered with $\left.\prec\right)$. Let $Q(X)=\sum_{j} b_{j} X^{j}$ be the minimal polynomial of $L$. $Q$ does not vanish at 0 since $L$ is invertible and it satisfies $\sum_{j} b_{j}\left(M^{j} w\right)^{\alpha}=0$. We now consider $r \in\left(\lambda^{-d / 2}, \lambda^{d / 2}\right)$ such that $r \mu \lambda^{|\alpha| / 2} e^{-i \theta}$ is never a root of $Q$ for any $\theta \in \mathbb{R}$. Finally, let $F(z) w^{\alpha} \in \mathcal{E}_{\alpha, r, 0}$, so that $F(\lambda z)=r^{-1} \lambda^{-|\alpha| / 2} e^{i \theta} F(z)$ for some $\theta \in \mathbb{R}$. We set $P(X)=Q\left(r \lambda^{|\alpha| / 2} e^{-i \theta} X\right)$. Then

$$
P\left(C_{\psi}\right)\left(F(z) w^{\alpha}\right)=\sum_{j} b_{j}\left(r \lambda^{-|\alpha| / 2} e^{-i \theta}\right)^{j} \cdot\left(r^{-1} \lambda^{|\alpha| / 2} e^{i \theta}\right)^{j} F(z)\left(M^{j} w\right)^{\alpha}=0
$$


and $P(\mu) \neq 0$. This means that $\mathcal{E}_{\alpha, r, 0} \subset \bigcup_{P \in \mathcal{P}_{\mu}} \operatorname{ker}\left(P\left(C_{\psi}\right)\right)$ for any $\mu \in \mathbb{C}$. Theorem 4.5 and Corollary 4.9 allow us to conclude that $C_{\psi}$ is cyclic.

\section{Applications to general COMposition OpERATORS}

5.1. Introduction. As mentioned in the introduction of the paper, one of the reasons to introduce linear fractional composition operators was the hope to use them to deduce properties of composition operators associated to general holomorphic self-maps of the ball. This is called the transference principle, which has been proved to be very efficient in the one-dimensional setting. Here is one of the results that we may obtain on $\mathbb{D}$ (see $[6]$ ). Let $\phi: \mathbb{D} \rightarrow \mathbb{D}$ be holomorphic and satisfying the following properties:

(i) $\phi$ extends to a continuous and univalent self-map of $\overline{\mathbb{D}}$;

(ii) $\phi(\overline{\mathbb{D}} \backslash\{1\}) \subset \mathbb{D}$;

(iii) $\phi$ has Denjoy-Wolff point at +1 with $\phi^{\prime}(1) \in(0,1)$ and $\phi$ has $C^{1+\varepsilon}$-smoothness at +1 .

Then $C_{\phi}$ is hypercyclic.

The strategy applied by Bourdon and Shapiro to prove this result can be summarized as follows. They first build a linear-fractional model for $\phi$; namely, they identify two self-maps of $\mathbb{D}$, denoted by $\sigma$ and $\phi_{\lambda}$, such that $\phi_{\lambda}$ is a hyperbolic linear-fractional map of $\mathbb{D}$ and the intertwining equation $\sigma \circ \phi=\phi_{\lambda} \circ \sigma$ holds true.

Next, they transfer the hypercyclicity of $C_{\phi_{\lambda}}$ to $C_{\phi}$ via $C_{\sigma}$; precisely, if $f$ is any hypercyclic vector of $C_{\phi_{\lambda}}$, then $C_{\sigma}(f)$ is a hypercyclic vector of $C_{\phi}$.

A general theory of linear fractional models in $\mathbb{B}_{d}$ has been developed in [1] (see also [8]). However, two difficulties arise in the several-variables context when we try to imitate the last part of the proof. First, not every self-map of $\mathbb{B}_{d}$ induces a bounded composition operator on $H^{2}\left(\mathbb{B}_{d}\right)$. Second, because of the lack of Mergelyan's theorem in $\mathbb{C}^{d}$, it is rather difficult to prove that a composition operator $C_{\sigma}$ has dense range in $H^{2}\left(\mathbb{B}_{d}\right)$.

At this stage, we do not expect to be able to prove a theorem which is as general as the Bourdon and Shapiro theorem. However, we will show that a small perturbation of a hyperbolic linear-fractional map preserves the dynamical properties of the associated composition operator. Here is the main statement of this section.

Theorem 5.1. Let $\psi: \mathbb{H}_{d} \rightarrow \mathbb{H}_{d}$ be a holomorphic self-map of $\mathbb{H}_{d}$ which may be written as

$$
\psi(z, w)=(\lambda z+R(z), M w+c)=\left(\psi_{1}(z), \psi_{2}(w)\right)
$$

with

- $\psi_{1}$ extends continuously to a continuous and univalent self-map of $\overline{\mathbb{P}_{+}} \cup$ $\{\infty\}$

- $R(z)=o\left(|z|^{1-\varepsilon}\right), \varepsilon>0$, when $|z| \rightarrow+\infty, z \in \mathbb{P}_{+}$;

- $\lambda>1, \inf _{z \in \mathbb{P}_{+}} \Im m(R(z)) \geq d>0$;

- $Q=\lambda I-M M^{*}$ is a Hermitian positive semi-definite matrix;

- $d-\|c\|^{2}>\left\langle Q^{+} M^{*} c, M^{*} c\right\rangle$.

Suppose moreover that $C_{\psi}$ is a bounded operator on $\mathcal{H}^{2}\left(\mathbb{H}_{d}\right)$. Then $C_{\psi}$ is supercyclic on $\mathcal{H}^{2}\left(\mathbb{H}_{d}\right)$.

Let us comment on this theorem. The two first conditions are regularity properties which are required on $\psi_{1}$. They are already present in the one-variable setting. 
The last three conditions are here to ensure that $\psi$ is a hyperbolic self-map of $\mathbb{H}_{d}$. Finally, we have to add that $C_{\psi}$ defines a bounded composition operator on $\mathcal{H}^{2}\left(\mathbb{H}_{d}\right)$, since this is (in general) not automatic. If we had imposed more regularity on $\psi$ near $\infty$, we would obtain the continuity of $C_{\psi}$ by applying Wogen's theorem (see [24]).

Our proof that $C_{\psi}$ is supercyclic does not consist in the transference of a supercyclic vector. We will rather transfer the assumptions of the supercyclicity criterion. More precisely, we assume for a while that the following two claims are true:

Claim 1. There exists $\sigma=(E, D): \mathbb{H}_{d} \rightarrow \mathbb{H}_{d}$ and $b \in \mathbb{C}$ with $\Im m(b)>0$ so that

$$
\sigma \circ \psi=\psi_{\lambda, b, M, c} \circ \sigma
$$

with $\psi_{\lambda, b, M, c}(z, w)=(\lambda z+b, M w+c)$ a hyperbolic linear fractional map of $\mathbb{H}_{d}$.

Claim 2. For any $\alpha \in \mathbb{N}^{d-1}, C_{\sigma}$ maps $\mathcal{E}_{\alpha}$ continuously into $\mathcal{E}_{\alpha}$, and $C_{\sigma}\left(\mathcal{E}_{\alpha}\right)$ is dense in $\mathcal{E}_{\alpha}$.

Let us show how we can deduce from this that $C_{\psi}$ is supercyclic by applying the outer supercyclicity criterion. Let $r>\lambda^{-d / 2}$ and $\mathcal{F}_{\alpha, r, b}=C_{\sigma}\left(\mathcal{E}_{\alpha, r, b}\right)$. By Claim 2 and since $\mathcal{E}_{\alpha, r, b}$ is dense in $\mathcal{E}_{\alpha}$, the subset $\mathcal{Y}=\bigoplus_{\alpha \in \mathbb{N}^{d-1}} \mathcal{F}_{\alpha, r, b}$ is dense in $\mathcal{H}^{2}\left(\mathbb{H}_{d}\right)$. Let us fix $y=F \circ E(z, w) w^{\alpha} \in \mathcal{F}_{\alpha, r, b}$ and let us set

$$
\mathcal{Z}=\left\{\sum_{|\beta| \leq N} a_{\beta} F \circ E(z, w) w^{\beta} ; a_{\beta} \in \mathbb{C}\right\}
$$

with $|\alpha|=N$. Let us also consider $Z=\left\{\sum_{|\beta| \leq N} a_{\beta} F(z) w^{\beta}: a_{\beta} \in \mathbb{C}\right\}$ as in Section 4.4. The intertwining map $\sigma$ induces a commutative diagram

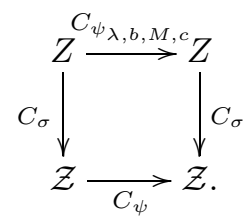

Since $Z$ and $\mathcal{Z}$ have finite dimension, and since $C_{\sigma}$ is one-to-one, it is an isomorphism from $Z$ onto $\mathcal{Z}$. Therefore, the work done in Section 4.4 carries on into this new context, and we can find a sequence $\left(S_{n} y\right)$ in $\mathcal{Z}$ and a positive constant $C$ such that $\left\|S_{n} y\right\| \leq C^{n}\|y\|$ and $C_{\psi}^{n} S_{n} y=y$.

The construction of $X_{y}$ follows the same idea. We shall prove that, for any $\gamma \in \mathbb{N}^{d-1}$, for $\rho:=\rho(\gamma, y)$ large enough, any vector $x$ of $\mathcal{F}_{\gamma, \rho, b}$, which is dense in $\mathcal{E}_{\gamma}$, will satisfy $\left\|S_{n} y\right\| \cdot\left\|C_{\psi}^{n} x\right\| \rightarrow 0$. The proof done above can be reproduced mutatis mutandis. We may then apply the outer supercyclicity criterion since $\bigoplus_{\gamma \in \mathbb{N}^{d-1}} \mathcal{F}_{\gamma, \rho, b}$ is dense in $\mathcal{H}^{2}\left(\mathbb{H}_{d}\right)$.

Therefore, our last task is to prove Claim 1 and Claim 2.

5.2. The intertwining map. We suppose that $\psi_{1}(z)=\lambda z+R(z)$ with $\inf (\Im m(R(z))) \geq d>0$ and $R(z)=o\left(|z|^{1-\varepsilon}\right)$ for some $\varepsilon>0$. We also assume that $\psi_{1}$ is continuous and univalent on $\overline{\mathbb{P}_{+}} \cup\{\infty\}$. Let us set $z(n)=\psi_{1}^{[n]}(z)=$ $\psi_{1} \circ \cdots \circ \psi_{1}(z)$. It is straightforward to observe that

$$
\frac{z(n)}{\lambda^{n}}=z+\sum_{j=0}^{n-1} \frac{R(z(j))}{\lambda^{j+1}}
$$


The work done in the proof of Theorem 4.7 in [6] shows that the series $\sum_{j=0}^{n-1} \frac{R(z(j))}{\lambda^{j+1}}$ converges uniformly on compact subsets of $\overline{\mathbb{P}_{+}}$to a holomorphic function $H$. The function $z \mapsto z+H(z)$ is continuous and univalent on $\overline{\mathbb{P}_{+}} \cup\{\infty\}$. Moreover, the imaginary part of $H$ is bounded below. Indeed,

$$
\Im m(H(z)) \geq \sum_{j=0}^{+\infty} \frac{d}{\lambda^{j+1}}=\frac{d}{\lambda-1}>0
$$

Let us set

$$
E(z)=z+H(z)-\frac{i \theta d}{\lambda-1}
$$

with $\theta \in(0,1)$. Then

$$
\begin{aligned}
E \circ \psi_{1}(z) & =\lim _{n \rightarrow+\infty} \frac{z(n+1)}{\lambda^{n}}-\frac{i \theta d}{\lambda-1} \\
& =\lambda\left(E+\frac{i \theta d}{\lambda-1}\right)-\frac{i \theta d}{\lambda-1} \\
& =\lambda E+i \theta d .
\end{aligned}
$$

Thus, if we set $D(z, w)=w, \sigma=(E, D)$ and $b=i \theta d$, then $\sigma$ is a self-map of $\mathbb{H}_{d}$ which satisfies $\sigma \circ \psi=\psi_{\lambda, M, b, c} \circ \sigma$. Moreover, if we choose $\theta$ sufficiently close to 0 , then $\Im m(b)-\|c\|^{2}>\left\langle Q^{+} M^{*} c, M^{*} c\right\rangle$, so that $\psi_{\lambda, M, b, c}$ becomes a linear fractional map of $\mathbb{H}_{d}$. Thus Claim 1 is proved. However, to prove Claim 2, we shall need several properties of $E$, which are summarized below:

(Q1): $E$ maps $\overline{\mathbb{P}_{+}}$into $\mathbb{P}_{+}$and is univalent on $\overline{\mathbb{P}_{+}} \cup\{\infty\}$.

(Q2): There exist two constants $C_{1}, C_{2}>0$ such that

$$
\forall z \in \overline{\mathbb{P}_{+}}, C_{1}|z+i| \leq|E(z)+i| \leq C_{2}|z+i| \text {. }
$$

Property (Q1) has already been proved above. Moreover, it implies that (Q2) is true on compact subsets of $\overline{\mathbb{P}_{+}}$. So we just need to prove this last property when $|z|$ is large enough. Now, since $|R(z)|=o\left(|z|^{1-\varepsilon}\right)$, for any $\mu>\lambda$, we can find $M>0$ such that, for any $z \in \mathbb{P}_{+}$with $|z| \geq M$,

$$
|z| \leq\left|\psi_{1}(z)\right| \leq \mu|z|
$$

We start with such a $z$ and we iterate $\psi_{1}$. We find $|z(j)| \leq \mu^{j}|z|$, so that

$$
|H(z)| \leq \sum_{j=0}^{+\infty} \frac{\mu^{j(1-\varepsilon)}|z|^{1-\varepsilon}}{\lambda^{j+1}} \leq C|z|^{1-\varepsilon}
$$

if $\mu^{1-\varepsilon}$ is smaller than $\lambda$. This gives immediately (Q2).

5.3. The intertwining composition operator. We conclude this section by the proof of Claim 2. In this context, it is easier to work directly in $\mathbb{B}_{d}$. So we set

$$
\mathcal{F}_{\alpha}=\left\{f(z) w^{\alpha} \in H^{2}\left(\mathbb{B}_{d}\right)\right\}=\left\{g \circ \sigma_{c}: g \in \mathcal{E}_{\alpha}\right\} .
$$

We have to prove that $C_{\sigma_{c}^{-1} \circ \sigma \circ \sigma_{c}}$ maps $\mathcal{F}_{\alpha}$ into itself continuously and with dense range. For notational simplicity, we denote by $\sigma$ the map $\sigma_{c}^{-1} \circ \sigma \circ \sigma_{c}$. However, its coordinate functions are now denoted by $\sigma_{1}$ and $\sigma_{2}$, so that

$$
\sigma_{1}(z, w)=\frac{E \circ \sigma_{c}(z, w)-i}{E \circ \sigma_{c}(z, w)+i} \text { and } \sigma_{2}(z, w)=\frac{2 i w}{(1-z)\left(E \circ \sigma_{c}(z, w)+i\right)}:=\frac{w}{G(z)} \text {. }
$$


Observe that $E \circ \sigma_{c}(z, w)$ depends only on $z$. Thus, for any $F(z, w)=f(z) w^{\alpha}$ in $\mathcal{F}_{\alpha}$,

$$
F \circ \sigma(z, w)=\frac{f \circ \sigma_{1}(z)}{G(z)^{|\alpha|}} w^{\alpha}
$$

can be written as $g(z) w^{\alpha}$. To compute the norm of $F \circ \sigma_{c}$ in $H^{2}\left(\mathbb{B}_{d}\right)$, we use the following formula (see [25, Lemma 1.10]):

$$
\|F \circ \sigma\|^{2}=C \int_{|z| \leq 1}\left(1-|z|^{2}\right)^{d-2} \int_{|w|^{2}=1-|z|^{2}}\left|f \circ \sigma_{1}(z)\right|^{2} \frac{\left|w^{\alpha}\right|^{2}}{|G(z)|^{2|\alpha|}} d m(w) d A(z) .
$$

Now, it is easy to show that $G=\left(E \circ \sigma_{c}+i\right) /\left(\sigma_{c}+i\right)$, so that, by (Q2), there exists some $C>0$ such that

$$
\frac{1}{|G(z)|^{|\alpha|}} \leq C \text { for any } z \in \mathbb{D} \text {. }
$$

We then find

$$
\begin{aligned}
\|F \circ \sigma\|^{2} & \lesssim \int_{|z| \leq 1}\left(1-|z|^{2}\right)^{d-2} \int_{|w|^{2}=1-|z|^{2}}\left|f \circ \sigma_{1}(z)\right|^{2}\left|w^{\alpha}\right|^{2} d m(w) d A(z) \\
& \lesssim \int_{|z| \leq 1}\left(1-|z|^{2}\right)^{d-2+|\alpha|+(d-1) / 2}\left|f \circ \sigma_{1}(z)\right|^{2} d A(z) .
\end{aligned}
$$

Now, $\sigma_{1}$ is a self-map of $\mathbb{D}$, so it defines a continuous operator on the Bergman space $A_{d-2+|\alpha|+(d-1) / 2}(\mathbb{D})$. Hence,

$$
\begin{aligned}
\|F \circ \sigma\|^{2} & \lesssim \int_{|z| \leq 1}\left(1-|z|^{2}\right)^{d-2+|\alpha|+(d-1) / 2}|f(z)|^{2} d A(z) \\
& \lesssim \int_{|z| \leq 1}\left(1-|z|^{2}\right)^{d-2}|f(z)|^{2} \int_{|w|^{2}=1-|z|^{2}}\left|w^{\alpha}\right|^{2} d m(w) d A(z) \\
& \lesssim\|F\|^{2} .
\end{aligned}
$$

Let us now prove that $C_{\sigma}\left(\mathcal{F}_{\alpha}\right)$ is dense in $\mathcal{F}_{\alpha}$. More precisely, we shall prove that $\left\{\sigma_{1}^{p} \sigma_{2}^{\alpha}: p \geq 0\right\}$ is dense in $\mathcal{F}_{\alpha}$. Let $z^{m} w^{\alpha} \in \mathcal{F}_{\alpha}$ and let us approximate it by a finite linear fractional combination $F(z)=\sum_{p \geq 0} a_{p} \sigma_{1}^{p}(z) \sigma_{2}^{\alpha}(z, w)$. Using the same formula, we infer that

$$
\begin{aligned}
& \left\|F-z^{m} w^{\alpha}\right\|^{2} \\
& \lesssim \int_{|z| \leq 1}\left(1-|z|^{2}\right)^{d-2} \int_{|w|^{2}=1-|z|^{2}}\left|\sum_{p \geq 0} a_{p} \sigma_{1}^{p}(z)-G(z)^{|\alpha|} z^{m}\right|^{2} \frac{\left|w^{\alpha}\right|^{2}}{|G(z)|^{2|\alpha|}} d m(w) d A(z) \\
& \lesssim \int_{|z| \leq 1}\left(1-|z|^{2}\right)^{d-2+|\alpha|+(d-1) / 2}\left|\sum_{p \geq 0} a_{p} \sigma_{1}^{p}(z)-G(z)^{|\alpha|} z^{m}\right|^{2} d A(z) .
\end{aligned}
$$

The proof follows because $\sigma_{1}$ is a univalent and continuous self-map of $\overline{\mathbb{D}}$. By Walsh's theorem, the polynomials are dense in the algebra $A\left(\sigma_{1}(\mathbb{D})\right)$. This in turn implies the polynomials in $\sigma_{1}$ are dense in the Bergman space $A_{d-2+|\alpha|+(d-1) / 2}(\mathbb{D})$. Observe that this $G$ is bounded and the function $z \mapsto G(z)^{|\alpha|} z^{m}$ belongs to this space. 
5.4. Conclusion. We hope that Theorem 5.1 could be extended to a more general context. As mentioned before, at least on $\mathbb{B}_{2}$, a linear fractional model does exist for a wide class of self-maps of $\mathbb{B}_{2}$. Assuming some regularity on $\psi$ near $\infty$, it is reasonable to expect that the transfer map $\sigma$ will have some regularity near $\infty$. We then can hope to apply Wogen's theorem to ensure that $C_{\sigma}$ defines a continuous operator on $\mathcal{H}^{2}\left(\mathbb{H}_{d}\right)$. The main problem seems to be how to prove that $C_{\sigma}$ has dense range. A famous example of Wermer 23. points out that a domain can be biholomorphic to the bidisk without being a Runge domain (the polynomials are not dense in $H(G)$ ). That was the main reason to restrict ourselves to a map $\psi=\left(\psi_{1}, \psi_{2}\right)$, where $\psi_{1}$ just depends on $z$ and $\psi_{2}$ just depends on $w$.

\section{REFERENCES}

[1] F. Bayart, The linear fractional model on the ball, Rev. Mat. Iberoamericana 24 (2008), 765-824. MR2490162(2010a:32032)

[2] F. Bayart, Parabolic composition operators on the ball, Adv. Math. 223 (2010), 1666-1705. MR2592506 (2011f:47033)

[3] F. Bayart, É. Matheron, Dynamics of Linear Operators, Cambridge Tracts in Math, vol. 179, Cambridge University Press, 2009. MR 2533318 (2010m:47001)

[4] L. Bernal-González, A. Bonilla, M. Calderón-Moreno, Compositional hypercyclicity equals supercyclicity, Houston J. Math 33 (2007), 581-591. MR2308997 (2008c:47015)

[5] C. Bisi, F. Bracci, Linear fractional maps of the unit ball: a geometric study, Adv. Math. 167 (2002), 265-287. MR 1906258 (2003d:32013)

[6] P. Bourdon, J. Shapiro, Cyclic phenomena for composition operators, Mem. Amer. Math. Soc. 596 (1997). MR:1396955 (97h:47023)

[7] F. Bracci, M. D. Contreras, S. Diaz-Madrigal, Classification of semi-groups of linear fractional maps in the unit ball, Adv. Math. 208 (2007) 318-350. MR.2304320 (2008e:32023)

[8] F. Bracci, G. Gentili, Solving the Schröder equation at the boundary in several variables, Michigan Math J. 53 (2005), 337-356. MR2152705 (2006e:32018)

[9] X. Chen, G. Cao, K. Guo, Inner functions and cyclic composition operators on $H^{2}\left(B_{N}\right), J$. Math. Anal. Appl. 250 (2000), 660-669. MR1786088 (2001g:47040)

[10] C. Cowen, Composition operators on $H^{2}$, J. Operator Theory 9 (1983) 77-106. MR695941 (84d:47038)

[11] C. Cowen, B. MacCluer, Composition Operators on Spaces of Analytic Functions, Stud. Adv. Math., CRC Press, 1995. MR.1397026 (97i:47056)

[12] C. Cowen, B. MacCluer, Linear fractional maps of the ball and their composition operators, Acta. Sci. Math (Szeged) 66 (2000), 351-376. MR.1768872 (2001g:47041)

[13] P. Düren, Theory of $H^{p}$ spaces, Academic Press, 1970. MR0268655 (42:3552)

[14] N. S. Feldman, V. G. Miller, T. L. Miller, Hypercyclic and supercyclic cohyponormal operators, Acta. Sci. Math (Szeged) 68 (2002), 965-990. MR.1954557(2004d:47021b)

[15] E. Gallardo-Gutiérrez, A. Montes-Rodríguez, The role of the spectrum in the cyclic behavior of composition operators, Mem. Amer. Math. Soc. 791 (2004). MR2023381 (2004k:47050)

[16] L. Jiang, C. Ouyang, Cyclic behavior of linear fractional composition operators on $H^{2}\left(B_{n}\right)$, J. Math. Anal. Appl. 341 (2008) 601-612. MR2394108 (2009a:47016)

[17] M. Jury, Norms and spectral radii of linear fractional composition operators on the ball, $J$. Funct. Anal. 254 (2008) 2387-2400. MR2409166 (2009d:47021)

[18] B. MacCluer, Spectra of automorphism-induced composition operators on $H^{p}\left(B_{N}\right)$, J. London Math. Soc. (2), 30 (1984), 95-104. MR760878 (86g:47036)

[19] E. Nordgren, Composition operators, Canad. J. Math., 20 (1968), 442-449. MR0223914 $(36: 6961)$

[20] W. Rudin, Function theory in the unit ball of $\mathbb{C}^{n}$, Die Grundlehren der Mathematischen Wissenschaften 241 (Springer, Berlin, 1980). MR601594 (82i:32002)

[21] J. Shapiro, Composition Operators and Classical Function Theory, Springer, 1991. MR:1237406 (94k:47049)

[22] M. Taniguchi, Chaotic composition operators on the classical holomorphic spaces, Complex Variables Theory Appl. 49 (2004), 429-538. MR2088044 (2006c:47030) 
[23] J. Wermer, On a domain equivalent to the bidisk, Math. Ann. 248 (1980), 193-194. MR:575937 (81g:32002)

[24] W. Wogen, The smooth mappings which preserve the Hardy space $H^{2}\left(\mathbb{B}_{n}\right)$, Oper. Theory Adv. Appl. 35 249-267, Birkhäuser, 1988. MR1017673 (90k:32018)

[25] K. Zhu, Spaces of holomorphic functions in the unit ball, Graduate Texts in Math. 226, Springer-Verlag, 2005. MR2115155 (2006d:46035)

Laboratoire de Mathématiques, Clermont Université, Université Blaise Pascal, BP 10448, F-63000 CLERMONT-FERRAND, CNRS, UMR 6620, Laboratoire de MathÉmatiques, F-63177 Aubiere, France

E-mail address: Frederic.Bayart@math.univ-bpclermont.fr

Laboratoire de Mathématiques, Université Paris-Sud, BÂtiment 425, 91405 Orsay, FRANCE

E-mail address: stephane.charpentier@math.u-psud.fr

Current address: Centre de Mathématiques et Informatique (CMI), Aix-Marseille Université, Technopôle Château-Gombert, 39, rue F. Joliot Curie, 13453 Marseille Cedex 13, France

E-mail address: stecharp@gmail.com 